\title{
8 Asymmetrische Interdependenz: Das regionale Entwicklungsprogramm TIRDEP in Tanga
}

Mradi, Swahili für „Intention“ wurde in den Jahrzehnten nach der Unabhängkeit auch die übliche Übersetzung für „Projekt“ im Sinne größerer Investitionsvorhaben und Entwicklungsprojekte. Etwa ab Ende der 1970er-Jahre erweiterte sich der semantische Bezugsraum noch weiter, sodass der Begriff mradi auch zunehmend private Maßnahmen zur Einkommensgenerierung umfasste, also solche „Projekte“ (Mehrzahl miradi), wie sie alle staatlichen Angestellten und wohl die gesamte urbane Bevölkerung in Tansania in den 1980er-Jahren verfolgen mussten, um den Einkommensverlust durch den gesunkenen Reallohn zu kompensieren. ${ }^{1}$ Projekte der „Technischen Hilfe“ mussten sich an diese Umstände - die konkreten Wirtschaftsbedingungen und Lebensrealitäten in Tansania - anpassen, zumal die Entwicklungsarbeit längst integraler Teil persönlicher Karriereund Überlebensstrategien geworden war. ${ }^{2}$

Dieses Kapitel diskutiert die Zusammenhänge von Akkumulationsstrategien, Disziplinierungspraktiken und Ressourcenallokation im Spiegel entwicklungspolitischer Trends und der Counterpartbeziehungen in TIRDEP. TIRDEP steht als Kürzel für ein GTZ-unterstützes regionales Entwicklungsprogramm in Tansania, das Tanga Integrated Rural Development Programme. Begonnen als Planungsvorhaben für nur einen Distrikt innerhalb der Region wurde das Programm auf die ganze Tanga-Region ausgeweitet und so „one of the largest and most ambitious German aid projects in the world, being in the forefront of the then widely advocated concept of ,integrated regional development programmes'“.3 TIRDEP bestand aus 31 Einzelprojekten in den verschiedensten Sektoren, die zwischen 1972 und 1991 (und in mehreren Fällen über das Ende des Programms hinaus) umgesetzt wurden. TIRDEP war ein Kind des „goldenen Zeitalters“ der entwicklungspolitischen Beziehungen zwischen der BRD und Tansania (siehe Kapitel 3). Im Zeitraum von achtzehn Jahren wandelten sich Schwerpunkte und Ziele des

1 Siehe Tripp, Changing the Rules, S. 171; Bryceson, Food, S. 214; Gundula Fischer, Unsere Fabrik - unsere Familie: Vorstellungen von Verwandtschaft und Familie in einem tansanischen Industriebetrieb vor der Privatisierung und danach, Berlin 2006, S. 81.

2 Melina Kalfelis, Flexibel aus Armut. Die Lebenswelt von lokalen Entwicklungsakteuren in Burkina Faso vor dem Hintergrund entwicklungspolitischer Erwartungshaltungen, in: Paideuma 61 (2015), S. 117-142; Green, The Development State.

3 Helmut Bley/Rolf Hofmeier: Director of the Institute of African Affairs, 1976-2000. A Retrospective, in: Ulf Engel u.a., Hg., Tanzania Revisited: Political Stability, Aid Dependency, and Development Constraints 2000, S. ix-xii, hier: S. ix.

๑ OpenAccess. ๑ 2021 Eric Burton, publiziert von De Gruyter. (cc) BY Dieses Werk ist lizenziert unter der Creative Commons Attribution 4.0 International Lizenz. https://doi.org/10.1515/9783110705621-011 
Programms in einer bemerkenswerten Dynamik. In den ersten zwei Jahren, 1973/ 74, fanden fast ausschließlich Planungsaktivitäten statt, ab Mitte der 1970er ging es in der feldinternen Sprache späterer Jahre erst um die „Hardware“ (Bau von Schulen, Brunnen, Straßen), ab Ende der 1970er dann um „Software“ (Personalschulung und Etablierung funktionierender Institutionen) und ab Ende der 1980er schließlich um „Prozesse“ (Projektfindung und -implementierung mit den „Zielgruppen“). ${ }^{4}$ Demenstprechend wandelte sich auch das Verständnis, wer an Planung und Implementierung beteiligt sein sollte, welchen Akteuren ein Subjektstatus, welchen nur ein Charakter als passiv Beteiligten zugesprochen wurde und wer in welcher Form an den Projektressourcen teilhaben durfte und sollte. Die mehrfach veränderte Vorgangsweise erklärt sich einerseits aus globalen Trends und Diskursverschiebungen in der Entwicklungspolitik, andererseits aber auch aus konkreten Aushandlungsprozessen in Tansania und Tanga selbst. Diese Trends und Verhältnisse wiederum wurden vom Projektpersonal, externen EvaluatorInnen und den tansanischen Behörden unterschiedlich wahrgenommen. Was andere Autoren für Oxfam und die Schweizer Entwicklungszusammenarbeit gezeigt haben, gilt auch für TIRDEP: Die Erfahrungen „vor Ort“ gingen der Formulierung neuer Richtlinien oft voraus; die Interaktionen in der Peripherie wirkten auf die Denk- und Handlungsweisen im Zentrum zurück. ${ }^{5}$ Auf Projektebene gesehen lässt sich ebenfalls argumentieren, dass die heterogenen „Zielgruppen“ und „das Projekt“ sich gegenseitig beeinflussten. ${ }^{6}$ Die Modi der Ressourcenverteilung änderten sich ebenfalls aufgrund äußerer Bedingungen, gewandelter Sichtweisen und der Aushandlungsprozesse zwischen verschiedenen Parteien.

Zeitlich fällt das Programm TIRDEP genau in jene Zeit, in der es für TansanierInnen immer schwieriger wurde, mit ihrem regulären Gehalt den Lebensunterhalt zu sichern. Das gilt auch für die tansanischen BeamtInnen, die den Einzelprojekten des Programmes als Counterparts zugeordnet waren. Obwohl zunehmend unterwandert, war der leadership code der Deklaration von Arusha aus dem Jahr 1967, der Nebenverdienste verbot, immer noch in Kraft und betraf in

4 BMZ, Country Case Study „TIRDEP, Tanzania Summary Ex-Post Evaluation - Sustainability of Regional Rural Development Programmes (RRD), Bonn 2005, S. 12.

5 Jennings, Surrogates of the State; Lukas Meier, Die Macht des Empfängers. Gesundheit als Verhandlungsgegenstand zwischen der Schweiz und Tansania, 1970-1980, in: Sara Elmer u. a., Hg., Handlungsfeld Entwicklung. Schweizer Erwartungen und Erfahrungen in der Geschichte der Entwicklungsarbeit, Basel 2014, S. 125-144.

6 Lars Johansson, Ten Million Trees Later. Land Use Change in the West Usambara Mountains the Soil Erosion Control and Agroforestry Project in Lushoto District 1981-2000, Eschborn, Germany 2001, S. 62. Siehe hierzu auch van Beusekom, Negotiating Development. 
den 1980er- Jahren alle Staatsbediensteten. ${ }^{7}$ Trotz zu dieser Zeit bereits längst geduldeter und sogar geförderter Ausnahmen wie Viehzucht und anderer landwirtschaftlicher Aktivitäten im familiären Rahmen waren die Aneignungsmöglichkeiten, die ein Programm wie TIRDEP bot, nicht nur willkommen, sondern oft auch notwendig zum Erhalt eines urbanen Lebensstils und der Versorgung von Verwandten und weiteren Netzwerken.

Dabei deckten sich die Vorstellungen von Arbeitsdisziplin und gerechten Arbeitsverhältnissen auf tansanischer Seite nicht immer mit jenen der Geberorganisationen. Wie Victoria Bernal an einem Beispiel aus dem kolonialen Sudan zeigt und auch für postkoloniale Interventionen für zutreffend hält, sind Entwicklungsprojekte nicht primär ökonomische, sondern in erster Linie disziplinarische Arrangements: „disciplinary institutions that establish authority, encode moralities, and order social relations“. ${ }^{8}$ Fragen der Disziplin und der Machtunterschiede sind aber eng verknüpft mit materieller Ungleichheit, Machtverhältnissen und Extraversionsstrategien. Entwicklungsprojekte lassen sich daher auch als „exterritoriale Zone mit Sicherheitszaun“ fassen: Die „Zäune“ sollen in erster Linie verhindern, dass die Projektressourcen im chronisch unterfinanzierten Umfeld und klientelistischen Kanälen versickerten. ${ }^{9}$ Anders als die meisten Berater und UniversitätsdozentInnen, deren Erfahrungen in den vorherigen Fallstudien geschildert wurden, hatten expatriates und Counterparts in TIRDEP tagtäglich Umgang mit Fragen der Ressourcenallokation und Disziplinierung. Wie der Anthropologe Noel Dyck für disziplinarische Arrangements allgemein festhält, sind diese dann am erfolgreichsten, wenn sie ausgehandelt werden: „[D]iscipline is most successful when it emerges from a consensus or negotiated arrangement between those who impose it and those who submit to it". ${ }^{10}$ Das gilt auch, wie David Mosse gezeigt hat, für Entwicklungsprogramme: „governance brought by development schemes cannot be imposed; it requires collaboration and compromise“. ${ }^{11}$ Im Falle von TIRDEP umfassten die Ordnungs- und Disziplinierungsmechanismen nicht nur verhaltensstrukturierende Pläne, die

\section{Siehe Kap. 2.}

8 Victoria Bernal, Colonial Moral Economy and the Discipline of Development: The Gezira Scheme and „Modern“ Sudan, in: Cultural Anthropology 12/4 (1997), S. 447-449, hier: S. 449.

9 Holger Illi, Development Experts at the Interface: An Inquiry into the Knowledge Dimension of Allocative and Strategic Action in the Arena of a German/Nepalese Health Development Project, Research Report, Bielefeld 2001, S. 71.

10 Noel Dyck, Anthropological Perspectives on Discipline: An Introduction to the Issues, in: Noel Dyck, Hg., Exploring Regimes of Discipline: The Dynamics of Restraint, New York, Oxford 2008, S. 1-22, hier: S. 10.

11 Mosse, Cultivating Development, S. 7; siehe auch Li, The Will to Improve; Van Beusekom, Negotiating Development. 
Überwachung entlang hierarchischer Ketten und das Aufstellen von Regeln, sondern zunehmend auch materielle Anreize und Kompensationen - also eine Ökonomisierung der Arbeitsbeziehungen. Diese vollzog sich im Rahmen entwicklungspolitischer Paradigmenwechsel, ohne aber, wie hier argumentiert wird, von diesen Paradigmenwechseln voll determiniert zu werden.

In historischer Sicht lassen sich vier miteinander verflochtene Transformationen herausarbeiten, die über den Fall von TIRDEP hinausgehend relevant sind. Erstens verloren die Geber das - ohnehin nie übermäßig, aber in Tansania zeitweise doch ausgeprägte - Vertrauen in die administrativen und exekutiven Fähigkeiten staatlicher Institutionen und problematisierten zunehmend, dass die jeweiligen Polit- und Verwaltungseliten auf Dorf-, Distrikt-, Regional- und nationaler Ebene im Widerspruch zur proklamierten Politik nicht den Interessen der „Zielgruppen“ entsprechend handelten. Das führte, zweitens, zu einer Abkehr von kapitalintensiven Projektansätzen hin zu Maßnahmen, die auf die Stärkung öffentlicher Institutionen abzielten. Diese Maßnahmen, feldintern als capacitybuilding bezeichnet, blieben auf die Stärkung des staatlichen Entwicklungsprojekts ausgerichtet, wurden allerdings - drittens - zunehmend komplementiert und teils abgelöst von der Strategie, staatliche und lokale Eliten zu umgehen und mit „partizipativen“ Methoden einen direkten Zugriff auf die „Zielgruppen“ zu bekommen, um diese in Entscheidungen, Planungen und Umsetzung einzubeziehen. Konzeptuelle Ansätze dazu stellte nicht zuletzt der tansanische self-reliance-Diskurs bereit, auf den sich westdeutsche Achtundsechziger mit ihrer antikapitalistischen Entwicklungshilfekritik auch wiederholt beriefen. Die praktische Bedeutung des Partizipationsparadigmas erweist sich aber weniger als Resultat einer in den den kapitalistischen Zentren ausgedachten Strategie, die dann einfach in die Peripherie exportiert wurde, sondern mehr als Reaktion auf Widerstände im Umgang mit Behörden, die unmögliche vollständige Überwachung des Personals mittlerer Ebene und die immer wieder wahrgenommene „Unwilligkeit“ der „Zielgruppen“ zu den geberunterstützten Projekten eigene Beiträge (,Selbsthilfe“) zu leisten. Viertens ist diese Entwicklung gebunden an die Zurückdrängung egalitaristischer Prinzipien und des Anspruchs eines Wohlfahrtstaates zugunsten von „Eigenverantwortung“: Die Einführung dörflicher Selbstverwaltung mit Kostenbeteiligung wurde nun durch Projekte vorangetrieben, selbst in Bereichen wie der Wasserversorgung, in denen Regierung und Partei in einem noch unentschiedenen Machtkampf standen (siehe Kapitel 2).

Die vier dargestellten Transformationen sind aufgrund der komplexen Akteurskonstellationen und dynamischer Kontexte nicht linear und eindeutig, sondern widersprüchlich und inkonsistent. Die Handlungsspielräume und Aneignungsmöglichkeiten für die beteiligten AkteurInnen werden im Folgenden durch drei Prismen behandelt: erstens, durch einen Vergleich der Tätigkeit in 
geberunterstützten Projekten einerseits und der staatlichen Bürokratie im andererseits im Lichte der Biografien tansanischer Counterparts sowie von GTZ-ExpertInnen; zweitens, durch die Analyse der asymmetrischen, aber interdependenten Verhältnisse zwischen GTZ-expatriates und Counterparts; sowie drittens, durch Fragen der Disziplinierung auf verschiedenen Ebenen der Projekte und im Lichte des neuen Paradigmas der Partizipation.

\subsection{Das TIRDEP-Personal}

Tangas Straßen wurden in der ersten Hälfte der 1980er-Jahre wenig beansprucht, nur selten waren Lastwagen, Autos und Motorräder auf den Verkehrsadern der Region zu sehen. Der Import von Fahrzeugen war umständlich und für alle mit Ausnahme außer einer winzigen Elite unleistbar, Benzin und Ersatzteile waren schwer zu bekommen; selbst Fahrräder waren ein knappes und begehrtes Importgut. Bei den wenigen Gefährten, die vereinzelt auf den Straßen fuhren, war auffällig, dass bei den meisten das Kürzel TX auf dem Nummernschild prangte. ${ }^{12}$ TX stand für Tanzania Expatriate und kennzeichnete das Fahrzeug als ausländischen Besitz, weswegen diese Kennzeichnung zwischen „Gebern“ und „Partnern“ immer wieder ausgehandelt werden musste ${ }^{13}$ : Wer hatte das letzte Wort, wenn es um die Verwendung dieser knappen Ressource ging? Die Bezeichnung TX fand Eingang in den Alltagssprachgebrauch und Liedtexte: als Bezeichnung für expatriates, aber auch als Qualität der expatriateness, die Dingen und Personen gleichermaßen zu eigen sein konnte und spätestens in den 1980er-Jahren, als sich der Nationalisierungseifer der vorherigen zwei Dekaden abgenutzt hatte, meist positiv bewertet wurde. ${ }^{14}$ Die expatriateness färbte auf die NutzerInnen der Gefährte ab: selbst tansanische Arbeitskräfte, die auf Motorrädern mit TX-Nummernschildern durch Tanga fuhren, wurden bisweilen $T X$ genannt.

In Tanga gehörten viele dieser importierten Fahrzeuge zu TIRDEP-Projekten. Die ökonomische Bedeutung von TIRDEP in der Tanga-Region lässt sich eher an relativen als an absoluten Zahlen veranschaulichen: 1979 induzierte das Programm 57 Prozent der veranschlagten Mittel in das Entwicklungsbudget der

12 Tanga Library, AG 5-23, A. Kater, Report of a Visit to the Tanga Region, (16-30 April 1986), Den Haag, Mai 1986, S. 13; Interview \#50, Tansanischer Beamter und Community DevelopmentExperte.

13 Privatarchiv S. R., [BMZ-Evaluierung 1991 + TIRDEP Selbstevaluierung], Minutes of a meeting held on 25.4.1990, o.O. [Lushoto], o.D.

14 Askew, Performing the Nation, S. 314-315. In einem Liedtext von Othman Soud wird auch ein tansanischer Arzt mit einem Studienabschluss aus Deutschland als TX bezeichnet. 
Region (also den Haushalt, aus dem Projekte und Interventionen finanziert wurden, die nicht zu den laufenden Kosten zählten); vom gesamten Regionalbudget stellte TIRDEP 1979 damit runde 14 Prozent. ${ }^{15}$ Die Betonung der materiellen Präsenz der BRD darf aber nicht dazu verleiten, die tansanischen Beiträge zu übersehen, denn die Projekttätigkeiten banden auch die verschiedensten Ressourcen der „Empfängerseite“. Finanzielle Beteiligungen, administrative Kapazitäten und vor allem Fachkräfte wurden vertraglich als „Counterpartleistungen“ fixiert und von westdeutscher Seite mehr oder weniger strikt eingefordert. Im Falle eines Landwirtschaftsprojekts etwa verpflichtete sich Tansania, 24 mittlere und hohe FunktionärInnen sowie 135 field officers für Projektzwecke zu stellen. ${ }^{16}$ In vielen Fällen taten die Behörden ihr bestes, die Vereinbarungen zu erfüllen. Wenn sie die Projekte aber „als zusätzliche Aufgabe empfanden“ oder keinerlei freie Kapazitäten hatten, wurde auch kein tansanisches Personal zur Verfügung gestellt. $^{17}$

Neben TX waren auch TIRDEP und die GTZ in Tanga und darüberhinaus schnell zu prestigereichen Markennamen geworden. Eine Anstellung bei der GTZ war ein „trademark“ im Lebenslauf und öffnete Türen in der weiteren Karriere, „people are thinking you are somebody“, wie zwei tansanische Counterparts im Interview versicherten. ${ }^{18}$ Hinter dem symbolischen Kapital, das aus einer persönlichen Assoziierung mit TX, TIRDEP und GTZ resultierte, stand nicht nur die wirtschaftliche Potenz der westlichen Entwicklungshilfe. Viele TansanierInnen drückten im Interview ihren Stolz aus, in einem TIRDEP-Projekt bzw. für die GTZ gearbeitet, die eigenen Fähigkeiten entwickelt und zum Nutzen der Bevölkerung angewendet zu haben. ${ }^{19}$ Allerdings hatten längst nicht alle, die in bzw. mit TIRDEP-Projekten arbeiteten, die Intention gehabt, in Tanga zu leben. Hier spielten die Imperative des Entwicklungsstaates und deren Einfluss auf individuelle Lebenswege eine zentrale Rolle.

15 Pilgram/Zils, TIRDEP, S. 86-87. Dabei fielen die von der BRD übernommenen Kosten, wie üblich, nur zum Teil tatsächlich im „Partnerland“ an, nur etwa $60 \%$ der zugesagten Mittel kamen in der tansanischen Wirtschaft an. Viele Waren wurden im Ausland bezogen, die Gehälter der GTZExpertInnen auf deutsche Konten überwiesen und nur im jeweils für notwendig erachteten Anteil von den Entsandten privat nach Tansania transferiert.

16 PAAA, ZW 116859, Arrangement for Agricultural Extension Project in TIRDEP, 31.10.1977.

17 Privatarchiv S. R., [KORRESPONDENZ MIT GTZ-HQ FB 221], H.-J. B., Abschlussbericht TIRDEP - Berater in Regional Ujamaa Aid Cooperative Development Department, Tanga, August 1983.

18 Interview \#85, Tansanischer Ingenieur und Counterpart; Interview \#84, Tansanischer Entwicklungsplaner.

19 Interview \#15, Tansanische GTZ-Expertin; Interview \#51, Tansanischer Techniker und Abteilungsleiter. 


\section{Wege nach Tanga}

Grundsätzlich muss unterschieden werden zwischen Personen, die direkt in Projekten angestellt waren, und jenen, die eigentlich tansanische BeamtInnen waren und zum Projekt sekundiert bzw. delegiert wurden. ${ }^{20}$ Als Richtlinie von TIRDEP galt im Interesse der Nachhaltigkeit, das Projektpersonal so weit wie möglich von staatlicher Seite gestellt zu bekommen. ${ }^{21}$ Allerdings war auf allen Ebenen der regionalen Bürokratie stets ein Mangel an Fachpersonal mit den benötigten Qualifikationen zu konstatieren. 1982 wurden für Tangas Behörden - bei insgesamt 12.533 Posten - 1.089 offene Stellen gelistet, mehr als in jeder anderen Region. ${ }^{22}$ Das Fachpersonal der mittleren und höheren Ebenen für TIRDEP wurde zumeist vonseiten tansanischer Behörden gestellt, während das Projekt Hilfspersonal (Fahrer, Sekretärinnen, Wachleute, Reinigungskräfte) und bisweilen auch Fachkräfte der mittleren Ebene direkt anstellte.

Die Hierarchie der Counterparts offenbarte sich insbesondere bei den Bildungsabschlüssen und dem Zugang zu weiteren Fortbildungsangeboten. Counterparts höherer Ebenen konnten in der Regel akademische Abschlüsse (degree) vorweisen, die teils aus westlichen Ländern oder auch kommunistischen Ländern wie der DDR stammten. Viele von ihnen hatten internationale Erfahrungen im Rahmen von Arbeitsbesuchen, Fortbildungen und Workshops gemacht. Das Fachpersonal der mittleren Ebene hatte in der Regel technische Ausbildungen (certificate, diploma) innerhalb Tansanias im Anschluss an die Sekundarstufe durchlaufen; einige konnten im Rahmen von meist geberfinanzierten Fortbildungen auch andere afrikanische Länder bereisen. Der Erhalt eines papiernen Nachweises - ob nun certificate, diploma oder ein prestigereicher degree - wurde in mehreren Interviews als der entscheidende biografische Moment des Übergangs zu einer neuen Rolle dargestellt: von diesem Zeitpunkt an fühlten und

20 Zwischen attachment und secondment bestehen ebenfalls Unterschiede; hier soll der Fokus aber auf der Differenz zwischen diesen beiden Formen einerseits und der direkten Anstellung andererseits liegen.

21 Dieses Prinzip war durchaus praxisrelevant: Siehe z. B. Tanga Library, Personnel Job Applications 1.2.7., VDP Project Manager an HEP Project Manager, o.O., o.D. [ca. 1989].

22 URT, Annual Manpower Report to the President 1982, Dar es Salaam 1984, S. 23 - 26. Unter den als offen angegebenen Stellen waren freilich auch Karteileichen und längst unnötig gewordene Posten, nichtsdestotrotz ist der Zahl ein gewisser Aussagewert zuzusprechen - auch wenn der Personalmangel mit dem Phänomen der Unterbeschäftigung koexistierte, das seinerseits v.a. durch Ressourcenmangel begründet war. Ein Fachkräftemangel bestand gerade in den höheren Positionen sowie in technischen und naturwissenschaftlichen Bereichen. 
präsentierten sich diese Personen legitim als mtaalam (Experte/Expertin) mit gesellschaftlich relevantem und statusverleihendem Spezialwissen. ${ }^{23}$

Im Laufe der 1970er-Jahre stellte der Staat zwar weiterhin mehr und mehr Personal ein, verlor aber sukzessive seinen noch aus der Kolonialzeit datierenden Nimbus als prestigereichster Arbeitgeber. Staatliche wie private Unternehmen zahlten nicht nur höhere Einstiegsgehälter, sondern boten auch umfassendere Lohnnebenleistungen (Unterkunft, Transport) und Aufstiegsmöglichkeiten. ${ }^{24}$ Ein Posten in der staatlichen Bürokratie hingegen war nach der Dezentralisierung 1972 mit Abstrichen in Status und Karriereaussichten verbunden. Das galt insbesondere für die Ministerialangestellten, die in die Regionalbehörden entsandt wurden und dort nicht nur weniger komfortable Arbeits- und Lebensbedingungen als in Dar es Salaam in Kauf nehmen mussten, sondern auch bei der Vergabe von Fortbildungsplätzen und Stipendien für Auslandssstudien oft übergangen wurden: „Consequently some of these officers have come to consider working in the regions to be a punishment“, berichtete das zuständige Ministerium $1976 .{ }^{25}$ Manche Personen weigerten sich gar, ihren Dienst in peripher gelegenen Städten oder ländlichen Gegenden anzutreten. ${ }^{26}$ Tanga war $\mathrm{u}$. a. aufgrund seiner Nähe zu Kenia im Vergleich mit anderen, infrastrukturell abgeschnittenen Regionen nicht das schlechteste Los, konnte aber mit der Attraktivität von Dar es Salaam oder Arusha nicht mithalten. ${ }^{27}$ Die zuständige Behörde sah in den vielen Klagen keinen Grund zur Sorge, solange die Personen immer noch entsprechend ihrer Qualifikation eingesetzt wurden. ${ }^{28}$

Interviews zeigen, dass eine Reihe tansanischer TIRDEP-Counterparts ungeplant oder gar unfreiwillig an ihrem Einsatzort in der Tanga-Region gelandet waren. Angesichts der materiellen Anreize und der symbolischen Präsenz der GTZ in Tanga - als wichtigste Geberinstitution geradezu omnipräsent - könnte man erwarten, dass Versetzungen in den Umkreis von TIRDEP-Projekten willkommen gewesen wären. In mehreren Gesprächen wurden allerdings konkrete biografische Gründe genannt, warum die Versetzung zu einem der GTZ-Projekte in Tanga kein Anlass zur Freude war. Ein Problem war der zwangsläufige Ortswechsel. Wichtig ist dabei ein Verständnis für einen idealtypischen Lebensentwurf in

23 Interview \#58, Tansanische Gartenbauexpertin; Interview \#53, Tansanischer Techniker.

24 URT, Manpower Report 1974, S. 41- 42.

25 URT, Manpower Report 1976, S. 9-10; siehe hierzu auch URT, Manpower Report 1977, Dar es Salaam 1982, S. $20-21$.

26 URT, Manpower Report 1976, S. 14.

27 Siehe z. B. Tanga Library, [Personnel Job Applications 1.2.7.], [unleserlich] an Project Manager VDP, Iringa, 28.11.1983.

28 URT, Manpower Report 1974, S. 41-42. 
Tansania, dessen Umsetzung gerade Angehörige der Bildungselite, aber auch andere Gruppen anstrebten. ${ }^{29}$ Sich „ein Leben aufzubauen“ (kujenga maisha) bedeutete in erster Linie materielle Absicherung und graduelle Verbesserung des Lebensstandards sowie, im Zusammenhang damit, die Erringung ökonomischer Eigenständigkeit und größere Entscheidungsautonomie gegenüber den älteren Generationen in der Familie. Etappen hierzu waren der Erwerb eines Stück Lands, der anschließende (sich oft über Jahre hinziehende) Bau eines „modernen“ Hauses aus Ziegeln mit einem Dach aus iron sheets und - ab Ende der 1970er Jahre auch gezwungenermaßen - die Diversifizierung der Einkommensbasis durch Aktivitäten in Landwirtschaft, Handel und Transport. Die Order von Vorgesetzten, den Tätigkeitsort zu wechseln, konnte bereits umgesetzten Bemühungen zur „Selbstentwicklung“ und dem Aufbau des eigenen Lebens einen herben Rückschlag versetzen. In dieser Art und Weise erinnerte sich ein Landwirtschaftsexperte an seine Versetzung innerhalb der Region Tanga von der Küstenstadt Tanga ins mehrere Stunden entfernte Lushoto in den Usambara-Bergen:

He told me that from the background of your courses, you have to go to Lushoto. At the beginning I refused to go. Because then I was working in Tanga. I had already my plot, I had already my farm in Pongwe. But again my boss thought that I could help a lot. [...] You know, sometimes if your boss asks you to do something, sometimes you don't have an alternative. Sometimes you have to agree. ${ }^{30}$

Der Vorgesetzte argumentierte, dass Personen mit dem entsprechenden Fachwissen dort wirksam werden sollten, wo sie gebraucht wurden. Die passende Qualifikation, meist symbolisch durch Bildungsabschlüsse ausgedrückt, wurde gemeinsam mit exzellenten Leistungen auch in anderen Interviews als Hauptgrund für Versetzungsentscheidungen und Beförderungen angegeben. ${ }^{31}$ Dieser für die Bürokratie so zentrale Mythos von der reinen Meritokratie und rationalistischen Entscheidungsfindung wurde in den meisten Interviews mit TansanierInnen bestätigt, nur in wenigen Fällen wurden persönliche Beziehungen (die westdeutsche GTZ-Entsandte häufig als tatsächlich ausschlaggebendes Motiv für Versetzungen und Beförderungen vermuteten) als ein Einflussfaktor genannt und dabei keinesfalls als Widerspruch zur Meritokratie gesehen. ${ }^{32}$ Ähnlichkeiten der

29 Maia Green hat die Strategien des kujenga maisha ausführlich für die Jahre ab 1990 dargestellt, siehe Green, The Development State, 50 - 51.

30 Interview \#80, Tansanischer Landwirtschaftsexperte.

31 Zum Beispiel Interview \#81, Tansanischer Bildungsexperte; Interivew \#90, Tansanischer Ingenieur und Counterpart.

32 Interview \#81, Tansanischer Entwicklungsplaner und Counterpart; Interview \#50, Tansanischer Beamter und Community Development-Experte. Zu komplementären und multiplen Logi- 
Personalpolitik mit dem kommunistischen Nomenklatur-System lassen sich kaum erkennen: Parteimitgliedschaft - oder politische Positionierung, darin waren sich alle Befragten einig, spielten keinerlei Rolle in der Selektion für diese Posten in Tanga; eine Parteimitgliedschaft wurde als reine Formalität dargestellt. Einige Tansanier mit DDR- und BRD-Studienabschlüssen wurden teils gegen ihren Willen von ihren Vorgesetzten mit der Annahme nach Tanga geschickt, dass sie aufgrund ihrer Überseeerfahrungen besonders gut mit „den Deutschen“ und ihrer strikten Art umgehen konnten. ${ }^{33}$

Allgemeiner Tenor in den Interviews war, dass Allokations- und Versetzungsentscheidungen (oft in der Fait accompli-Form eines Briefes übermittelt) alternativlos hingenommen werden mussten: „You just take your bag and leave. “34 Rationalisiert wurde die Alternativlosigkeit in Kategorien, die die individuelle Loyalität zum Entwicklungsstaat betonten und die Wohlfahrtsfunktion des Staates unterstrichen. Personalmangel und Personalplanung resultierten in der Pflicht, dem Staat, dem man seine Ausbildung verdankte, zur Verfügung zu stehen. Diese Variante des Sozialvertrags wurde auch in Interviews erwähnt und historisch der Ujamaa-Ära zugeordnet:

During this time there was an acute shortage of trained staff. So there was no way. You don't even have to apply. You are just given a letter, this is your letter, you are going to Arusha. You can't choose where you go. You just go. Because the government has paid for your training, everything. Tuition fees, boarding and lodging, everything. ${ }^{35}$

Die Verschränkung von offiziellen Diskursen des gleichzeitig gebenden wie rechtmäßig fordernden Entwicklungsstaates und den biografischen Narrativen ist augenfällig. Wie Pierre Bourdieu warnt, folgen biografische Erzählungen oft einer Offizialisierungsstrategie. Sie blenden also private (und damit in rationalistischen, bürokratischen Ordnungen illegitime) Interessen aus und stellen demgegenüber uneigennützige, dem Gemeinwohl förderliche Motive in den Vordergrund..$^{36}$ Der Verweis auf diese Ebene der Pflicht beantwortet jedoch nicht, warum

ken in afrikanischen Bürokratien siehe auch Thomas Bierschenk/Olivier de Sardan, Jean-Pierre, Studying the Dynamics of African Bureaucracies. An Introduction to States at Work, in: dies., Hg., States at Work: Dynamics of African Bureaucracies, Leiden, Boston 2014, S. 3-34.

33 Interview \#78, Tansanischer Landwirtschaftsexperte. Auch ein ehemaliger „Counterpart-Koordinator“ erwähnte, dass Tansanier mit Deutschkenntnissen bevorzugt nach Tanga geschickt wurden, um dort mit den GTZ-Personal zu arbeiten (Interview \#84).

34 Interview \#7, Tansanischer Entwicklungsplaner.

35 Ebd.; vgl. auch Interview \#90, Tansanischer Ingenieur und Counterpart.

36 Pierre Bourdieu, Die biographische Illusion, in: BIOS 3/1 (1990), S. 75-81, hier: S. 79. 
den Versetzungsanweisungen meist ohne Widerspruch Folge geleistet wurde. ${ }^{37}$ Ein entscheidender Grund ist die Opportunitätsstruktur, also der Mangel an alternativen Arbeitgebern für bestimmte Berufsgruppen, ein zweiter die Sicherheit der Beamtenstelle, zumindest ein geregeltes Einkommen zu haben, während die Quelle eines geberfinanzierten Projektes plötzlich versiegen konnte. ${ }^{38}$ Ein Techniker etwa legte in seiner Bewerbung für eine Stelle in einem TIRDEP-Projekt dar, dass ihn die Unterbrechung seiner Beamtenlaufbahn keine Sorgen bereite (,[I] [d] on't bother about interrupting of my civil service career“39), durchaus aber das Risiko, während der Probezeit entlassen zu werden: „Of course I have to safeguard my family well-fair [sic] just in case I will be eliminated“. ${ }^{40}$ Zudem war für jene Fachkräfte, die direkt durch das Projekt angestellt waren, ihre spätere Übernahme durch die Behörde nicht garantiert. ${ }^{41}$ Drittens konnte Widerspruch als Insubordination aufgefasst werden, was das Beziehungsklima mit Vorgesetzten und damit die eigene Karriere gefährdet hätte.

Familiäre Gründe spielten ebenfalls eine Rolle und konnten, um auf ein Konzept klassischer Migrationstheorien zurückzugreifen, als Pull- wie auch als Push-Faktoren wirken. Während für manche Fachkräfte die Nähe zu Familienmitgliedern einen essenziellen Bestandteil eines erfüllten Lebens darstellte, waren andere eher bemüht, die geografische Distanz zur Familie zu vergrößern. So sollte - umgekehrt proportional zur größer werdenden Distanz - die Wahrscheinlichkeit verringert werden, dass Verwandte mit Unterstützungsbedarf (engl. dependants, Kiswahili wategemezi) materielle Ressourcen einforderten, was den Aufbau des eigenen Lebens empfindlich bremsen konnte. ${ }^{42}$ Entsprechende Strategien räumlicher wie sozialer Distanzierung von Familienmitgliedern wurden

37 Als Gegenbeispiele lassen sich Beschwerdebriefe an die Parteiführung heranziehen, z.B. CCMA, THQ/A.25/3, R.F.M. an Katibu wa TANU wa Tanga, Chaki Chaki (Lushoto), 30.9.1974. Für TIRDEP im Speziellen ließen sich allerdings keine schriftlichen Belege dieser Art finden.

38 Interview \#85, Tansanischer Experte für Wasserversorgung.

39 Tanga Library, Personnel Job Applications 1.2.7, S. M. K. (Technician) an VDP Project Manager, Iringa, 30.3.1983.

40 Ebd., S. M. K. (Technician) an VDP Project Manager, Iringa, 11.4.1983.

41 Tanga Library, [General Correspondences], VDP Project Manager an RCDO, 13.11.1985; ebd., [RCDO], Minutes of a meeting with RCDO, 5.7.1990.

42 Interview \#51, Tansanischer Techniker und Abteilungsleiter; Interview \#58, Tansanische Gartenbauexpertin; Interview \#59, Tansanischer Landwirtschaftsexperte; Interview \#79, Tansanischer Experte in Community Development. Siehe auch die Bewerbungen in Tanga Library, „Personnel Job Applications 1.2.7“. 
während und auch schon vor der Ujamaa-Zeit verfolgt, um den eigenen Aufstieg zu gewährleisten und „auf eigenen Beinen stehen zu können“. ${ }^{43}$

Ein weiterer Faktor in der Bewertung des Arbeitsortes war die verbreitete Ansicht, dass es aus Statusgründen potenziell konfliktbringend und weniger effektiv sei, in der Herkunftsregion zu arbeiten. ${ }^{44}$ In den Worten einer Beamtin, die sich auf Landwirtschaftsfragen spezialisiert hatte:

Wenn du genau dort [in deiner Herkunftsregion] lebst, wirst du keine Beratung vornehmen können - du wirst es nicht schaffen, jemanden für neue Technologien zu ändern. Er/Sie wird sagen: „Du bist hier geboren, [...] was willst du uns schon erzählen?“ Aber wenn du an einem neuen Ort bist, als gänzlich Fremde, dann kannst du diese community verändern. ${ }^{45}$

Das Zitat unterläuft die simple Dichotomie von expatriates und nationalen Kadern auf eine andere Weise als auf die bisher erwähnten (eher strukturell begründeten): nämlich in Bezug auf Erfahrungen von Fremdheit im eigenen Land. Im bekannten Umfeld stoße man tendenziell häufiger auf Leute, die auf ihre Autorität (begründet z.B. auf Seniorität) pochen und damit stärkere Widerstände gegen jegliche Versuche der Verhaltensänderung hinnehmen müssen; entsprechende Befunde gibt es auch für die Region Tanga. ${ }^{46}$ Der Ortswechsel wird so zur Strategie, um sich einerseits von Kontrollansprüchen der Familie und Angehörigen der älteren Generationen zu lösen und andererseits als junge, gebildete Fachkraft mit eigenen Autoritätsansprüchen zu etablieren. ${ }^{47}$ Diese Strategien sind das Zeichen einer enormen Ungleichverteilung von Bildungskapital und dem damit einhergehenden Prestige. In einer Reihe von Fällen waren die tansanischen Counterparts und Angestellten in TIRDEP somit keine „locals“ im engeren Sinne, sondern mussten sich als impatriates ebenfalls erst auf die konkreten Gegebenheiten vor Ort einstellen. ${ }^{48}$

43 Vgl. hierzu die oft herangezogene Studie eines Soziologen, in der diese Strategie der sozialräumlichen Distanzierung ebenfalls beschrieben wird: J. A. K. Leslie, A Survey of Dar es Salaam, London 1963.

44 Interview \#78, Tansanischer Landwirtschaftsexperte.

45 Interview \#58, Tansanische Gartenbauexpertin. Das Zitat im Original: „Lakini ukikaa palepale, hutaweza kushauri kwa sababu - hautaweza kumchange yule mtu kwenye new technology. Atasema umezaliwa hapa, ... sasa unatuambia nini? Lakini unapokuwa sehemu nyingine, mtu mgeni kabisa, unaweza ukachange ile community.“

46 Tanga Library, TIR 1-3, URT, Tanga Regional Development Plan 1975-1980 Volume 2, Tanga, Mai 1975, S. 376. Die Ausführungen beziehen sich auf Forschungen von Michaela von Freyhold. 47 Das ist ein bekanntes Motiv in der afrikawissenschaftlichen Forschung, siehe z.B. Bjerk, Building a Peaceful Nation, 158-163.

48 Zum Begriff impatriates siehe Raymond Apthorpe, Who Is International Aid? Some Personal Observations, in: Anne-Meike Fechter/Heather Hindman, Hg., Inside the Everyday Lives of 
Ein entscheidender Nachteil für impatriates war, dass sie ähnlich den expatriates Probleme in Fragen wie der Unterkunftssuche hatten, ohne aber über deren Statusprivilegien und Finanzkraft zu verfügen. Auch sie mussten sich soziale Netzwerke und das Wissen in lokal spezifischen Fragen erst aufbauen, mitunter verspürten sie eine kulturelle Distanz und machten Alteritätserfahrungen. ${ }^{49}$ Ein tansanischer Experte aus Iringa (einer Region im Landesinneren), der in seiner Tätigkeit auf intensive Kommunikation angewiesen war, meinte zum Beispiel, dass er sich erst an das schnell gesprochene Kiswahili in der Küstenregion gewöhnen musste und den „Zielgruppen“ erst nach mehreren Fortbildungen effektiv Projektideen vermitteln konnte..$^{50}$ Da die tansanische Erfahrung allerdings ein absolutes Erfolgsbeispiel des nation-building darstellt, spielten ethnische Spannungen oder Sprachunterschiede keine signifikante Rolle in der Bewertung der Tätigkeit. Zentral waren in erster Linie sozioökonomische Fragen.

\section{Die „Firma“ TIRDEP als Arbeitgeber}

Viele ehemalige Angestellte und sekundierte Beamte der tansanischen Seite schilderten TIRDEP-Projekte in nostalgischen Farben als Sehnsuchtsorte reibungsloser Arbeitsaufläufe. Die diversen Schilderungen lassen sich zu folgender, zugespitzter Idealdarstellung amalgamieren: Alle MitarbeiterInnen hatten einen individuellen wöchentlichen Arbeitsplan, an den sie sich pflichtbewusst hielten. Sie konnten diese Pläne pflichtbewusst umsetzen, denn Autos und Motorräder waren stets einsatzbereit: gewartet, betankt (in Tanga-Mwambani und bei der Landwirtschaftsbehörde in den Usambarabergen hatte die GTZ sogar eine eigene, manchen Erzählungen zufolge niemals trockene Projekttankstelle eingerichtet, für deren Befüllung ein fässerbeladener Lastwagen regelmäßig zum Benzinkauf nach Kenia fuhr) und nach einem gemeinsam abgestimmten Plan zugeteilt. Jeden Freitag gab es eine Besprechung der Planerfüllung, in der alle MitarbeiterInnen ihr Programm für die nächste Woche vorstellten und die Zuweisung von Fahrzeugen, Werkzeugen und anderen Ressourcen miteinander abstimmten. Für den Tee mit Milch am Morgen war gesorgt, sodass sich niemand herausreden konnte, dass es kein Frühstück gab. Punkt 7.30 Uhr - Verspätungen gab es nicht - fuhren alle Autos ab. Zurück blieben nur die Sekretärin - und jene Angestellten und BeamtInnen, deren Abteilungen nicht in die Projektressourcenkreisläufe einbe-

Development Workers: The Challenges and Futures of Aidland, Boulder 2011, S. 193-210, hier: S. $207-208$

49 Siehe hierzu auch Bienen, Tanzania, S. 144.

50 Interview \#51, Tansanischer Techniker und Abteilungsleiter. 
zogen waren. Die Arbeit in oder mit TIRDEP, so lässt sich aus dieser Darstellung ableiten, war im Vergleich zum ,reinen“ Staatsdienst auch deshalb attraktiv, weil in den geberfinanzierten Projekten tatsächlich gearbeitet werden konnte, während im staatlichen Sektor die Ressourcenknappheit jeden Eifer lähmte. Die GTZ nahm mit ihren enormen Ressourceninputs, die dazu dienten, „die Counterpartbehörden auf einen funktionalen Normalstand anzuheben“, bewusst „in Kauf [...], dass damit ein starkes Gefälle zu anderen Fachbehörden“ entstand..$^{51}$ In vereinzelten Behörden, wo manche Abteilungen ins Projekt einbezogen waren und andere nicht, war dieses Gefälle an jedem einzelnen Morgen sichtbar: Während eine Hälfte des Personals motiviert mit vollbetankten Fahrzeugen ausschwärmte, blieb die andere mittellos und lethargisch in den Büros zurück. ${ }^{52}$

Die Darstellung der funktionierenden Hälfte ist gewissermaßen der Idealtyp des Projekts. Die Koexistenz funktionaler und dysfunktionaler Arbeitsabläufe, von Mobilität und Immobilität auf engstem Raum führt jedoch auch die Bedeutung der extern eingespeisten Ressourcen in Darstellungen von Arbeitsabläufen deutlich vor Augen. TIRDEP war einerseits ein Sprungbrett für soziale Mobilität, andererseits aufgrund der Statusunterschiede Aushandlungsort einer moralischen Mikro-Ökonomie. In den kühlen Berichten der GTZ-ExpertInnen nach Eschborn und noch mehr den informellen Korrespondenzen sind zahllose Beispiele von Missgeschicken, Verzögerungen, Planlosigkeit und unintendierten Nebeneffekten verzeichnet; auch in manchen Interviews mit TansanierInnen fanden Pannen und Probleme Erwähnung, wenngleich ihre Aussagen sich näher am Idealtyp bewegen. Wichtig ist daher zu beachten, vor welchem Erfahrungshintergrund die idealisierenden Aussagen gemacht wurden. Tatsächlich kontrastierten InterviewpartnerInnen die Arbeit in geberunterstützten Projekten üblicherweise mit Regierungsprojekten ohne externe Unterstützung, sprich: zwei konkret erfahrenen Kontexten. Wie oben bereits beschrieben, war es in Regierungspositionen aufgrund des Mangels an offiziellen Planvorgaben und Ressourcen oft unmöglich, die eigenen Aufgaben zu erfüllen und Erfüllung in der

51 BArch Koblenz, B 213/33065, Hildenbrand (GTZ), Arbeitspapier: Beurteilung der politischen Situation in Tansania und ihre Auswirkungen auf die durch die BRD geförderten Projekte, o.O., 27.1.1977, S. 7.

52 Dies war besonders der Fall bei SECAP in Lushoto, wo diese Koexistenz zum Teil extreme Züge annahm. Ein Teil der Behörde schwärmte mit dem Fuhrpark aus, der andere verblieb in den Büros, unfähig, die vorgesehenen Beratungstätigkeiten in den Dörfern durchzuführen. Interview \#58, Tansanische Gartenbauexpertin; Interview \#89, Schwedischer Volontär und Consultant. 
Tätigkeit zu finden. Einzelne TIRDEP-Projekte hatten zeitweise mehr Mittel zur Verfügung als die jeweils zuständige Distriktverwaltung. ${ }^{53}$

In mehreren Interviews wurde die Umsetzung langfristiger persönlicher Vorhaben mit den materiellen Gewinnen aus der Tätigkeit in TIRDEP verknüpft: „TIRDEP hat mir ermöglicht, mein Hüttchen, mein kleines Haus zu bauen, in dem ich heute lebe. “54 In manchen Projekten bekamen, was weder den allgemeinen entwicklungspolitischen Prinzipien der BRD noch konkreten Projektvereinbarungen entsprach, sekundierte Angestellte und Counterparts sogar Gehaltszuschläge. In materieller Hinsicht waren in den Jahren der Währungskrise nicht die Gehälter, sondern die Lohnnebenleistungen (marupurupu) das Entscheidende. Je nach Projekt konnten angestellte und sekundierte Fachkräfte damit rechnen, den Lohn - für sich genommen zum Überleben gerade in den frühen 1980er Jahren ungenügend - durch Tagessätze und Reisepauschalen (posho, ein Begriff, der bereits für die Tagesrationen im Karawanenhandel des ausgehenden 19. Jahrhunderts verwendet wurde) aufzubessern, Sonderzuteilungen von rationierten Grundnahrungsmitteln wie Reis, Zucker und Maismehl zu bekommen ${ }^{55}$ oder mit einem Projektkredit ein eigenes Motorrad oder Fahrrad anschaffen zu können. TIRDEP importierte viele lokal nicht erhältliche Güter wie Ersatzteile, Fahr- und Werkzeuge aus Kenia - was für Privatpersonen nicht möglich war.

Eine Anstellung in (oder Versetzung als Counterpart zu) TIRDEP bot daher unter Umständen Möglichkeiten, einkommensgenerierende „Projekte“ (miradi) privater Art $\mathrm{zu}$ initiieren oder $\mathrm{zu}$ vergrößern. Buchstäblich alle interviewten TansanierInnen verfolgten solche Einkommensstrategien, die über die Arbeit im Projekt hinausgingen und meist von Familienmitgliedern betrieben wurden: Handel mit Mais, Geflügel- und Rinderzucht, die Eröffnung einer Bar oder der Verkauf von maandazi (Krapfen), vitumbua (Reiskuchen) und anderen Snacks. Oft trugen diese meist von Frauen und Kindern betriebenen Aktivitäten deutlich mehr zum Familienhaushalt bei als das Einkommen aus dem Staatsdienst. ${ }^{56}$ Ein via TIRDEP erstandenes Fahrrad war eine Investition, die z.B. die Eröffnung eines

53 Pilgram/Zils, TIRDEP, S. 86-87. Dabei fielen die von der BRD übernommenen Kosten, wie üblich, nur zum Teil tatsächlich im „Partnerland“ an, nur etwa $60 \%$ der zugesagten Mittel kamen in der tansanischen Wirtschaft an. Viele Waren wurden im Ausland bezogen, die Gehälter der GTZExpertInnen auf deutsche Konten überwiesen und nur im jeweils für notwendig erachteten Anteil von den Entsandten privat nach Tansania transferiert.

54 Interview \#51, Tansanischer Techniker und Abteilungsleiter. Im Original: „TIRDEP kwangu mimi ilinifanya nijenge kakibanda kangu nyumba yangu ninamoishi leo.“

55 Tanga Library, [General Correspondences], VDP Project Manager an General Manager RTC, Tanga, 1.4.1986; ebd., VDP Project Manager an General Manager RTC, Tanga, 11.7.1990.

56 Tripp, Changing the Rules. 
Fahrradverleihs oder den Transport von Waren erlaubte und sich so vielfach rentieren konnte. ${ }^{57}$ Ähnlich erstand ein Counterpart während eines projektfinanzierten Fortbildungsaufenthaltes in England einen Kühlschrank, den dessen Ehefrau dann nutzte um Eis herzustellen und dieses in der Nachbarschaft zu verkaufen.

Die tansanischen Arbeitskräfte waren jedoch keinesfalls nur auf materielle Werte fixiert, sondern maßen auch einer sinnstiftenden, den eigenen Status- und Karrierevorstellungen entsprechenden Tätigkeit großen Wert bei. Wie oben bereits gezeigt, war das in geberunterstützten Projekten eher möglich als in der rein tansanisch finanzierten Bürokratie. Ein ehemaliger, aber rückkehrwilliger TIRDEP-Mitarbeiter schrieb in einer Bewerbung, dass er vor allem die Arbeitsauslastung und Verantwortung vermisste. ${ }^{58}$ Fachkräfte im öffentlichen Dienst beklagten sich in Initiativbewerbungen an TIRDEP darüber, dass ihr kulturelles Kapital degenerierte, weil es in ihrem Arbeitsalltag keine Anwendung fand:

[H]ere at Municipal Council I have been become so much bored with the endless hours of nothing to do, meaning that there is no building projects which are to be keeping me experienced and busy - and without taking this measure I would be diminishing my technology. ${ }^{59}$

BewerberInnen verbanden mit der „Firma“ (kampuni) TIRDEP, wie es in einem Bewerbungsschreiben hieß, die Möglichkeit, verantwortungsvollere Aufgaben entsprechend ihrer Qualifikation zu bekommen, weitere Bildungsangebote finanziert $\mathrm{zu}$ bekommen und Arbeitserfahrungen mit expatriates $\mathrm{zu}$ sammeln. ${ }^{60}$ Alle interviewten TansanierInnen stimmten darin überein, dass ein geberfinanziertes Projekt um ein Vielfaches mehr Möglichkeiten bot, Erfahrung und Expertise zu akkumulieren, als dies in staatlichen Posten ohne externe Unterstützung möglich war. Gerade für ExpertInnen auf einem fachlich hohen Niveau war die Präsenz von internationalen Fachkräften eine willkommene Möglichkeit, die eigene fachliche Exzellenz nicht nur unter Beweis zu stellen, sondern auch weiter zu fördern. So meinte ein Ingenieur, der sich selbst zur „cream of Tanzanians“ in seinem Fachbereich rechnete:

57 Interview \#57, Tansanischer Baufachmann.

58 „I was also used of having a big task means a lot to do while I am [lacking] sufficient work here.“ Tanga Library, Personnel Job Applications 1.2.7., [unleserlich] an Project Manager VDP, Iringa, 28.11.1983

59 Tanga Library, Personnel Job Applications 1.2.7, Bewerbungsschreiben G. K. an VDP Project Manager, 8.11.1989.

60 Ebd., Bewerbungsschreiben P. M. N. an TIRDEP Coordinator, Tanga, 13.2.1982; ebd., Bewerbungsschreiben K. M. an VDP Project Manager, 6.10.1989, o.0. 
I thought that was my privilege to explore and to show my technical competence. Because I was fully on irrigation, a seasoned irrigation engineer, so I thought that was also an opportunity. But also I looked forward to working with the experts. You know, we engineers, when we are trained, [if] you don't get someone to give you capacity, nobody to give you some training, you can't move forward. I was really pleased that I can now have contacts with some other well-experienced people. ${ }^{61}$

Die Aneignungsmöglichkeiten in TIRDEP-Projekten lassen sich dabei als eine auf den Kopf gestellte Pyramide denken: Je höher die Position eines Counterparts, desto wahrscheinlicher war der Zugang zu materiellen und immateriellen Projektressourcen. Am deutlichsten wird das beim kulturellen und symbolischen Kapital. Als Faustregel, für die es in TIRDEP viele Ausnahmen gibt, lässt sich festhalten, dass Counterparts umso längere und prestigereichere Fortbildungsmöglichkeiten wahrnehmen konnten, je höher ihre Position bereits war. „Uns Leuten von unten" (sisi watu wa chini), so erinnerte sich ein technischer Zeichner, blieben Aufstiegsmöglichkeiten durch Bildung verwehrt, obwohl entsprechende Forderungen wiederholt gestellt wurden und „wir viel Lärm gemacht haben“. ${ }^{62}$ Ein GTZ-Projektleiter erkannte ganz richtig das „top-heavy pattern of our scholarships": die Studienmöglichkeiten blieben dem tansanischen senior staff vorbehalten. ${ }^{63}$ Dem Masterstudium in England für einige höhere Counterparts standen mehrwöchige Seminare für MitarbeiterInnen der mittleren und unteren Ebenen gegenüber, in manchen Fällen gar nur informelles on-the-job training, das keinerlei symbolischen Wert für Gehaltseinstufungen oder zukünftige Bewerbungen aufwies. Bestehende Ungleichheiten wurden durch die Mobilitätspraktiken festgeschrieben oder noch weiter verschärft. ${ }^{64}$ Wie ein ehemaliger GTZ-Projektleiter ausführte, gehörte diese Bevorzugung $\mathrm{zu}$ den ungeschriebenen Spielregeln der Counterpartbeziehungen:

Wir haben auch nach Europa Leute geschickt, zu diesen Managementkursen, Projektmanagementkursen. Meinen Counterpart, die Spitzencounterparts sowieso immer. Das gehörte sich so. Da haben die sehr drauf geachtet, dass wer dieses Niveau erreicht hatte, OberCounterpart in einem Projekt zu sein, dass der auch mal in ein Auslandsprojekt reist. Weil das war ja damals sozusagen fast DDR-Niveau für die Leute, die Ausreise. Einen Pass zu

61 Interview \#85, Tansanischer Ingenieur und Counterpart.

62 Interview \#59, Tansanischer technischer Zeichner; im Original: „[...] tulipiga kelele sana“.

63 Tanga Library, TIR PAM 0060, VDP Project Manager, Project Progress Report January-June 1982, Tanga, 30.6.1982, S. 22; ebd., BMZ, Main Report on the inspection of the Technical Cooperation Project TIRDEP, Bonn, 1.12.1982, S. 66.

64 Für eine ausführliche Darstellung dieses Arguments siehe Rebecca Warne Peters, Development Mobilities: Identity and Authority in an Angolan Development Programme, in: Journal of Ethnic and Migration Studies 39/2 (2013), S. 277-293. 
kriegen, eine Ausreise zu kriegen. Sehr wichtig. War ja auch eine Art von Geschenk dann, Anerkennungsgeschenk. ${ }^{65}$

Für jene, die nicht in den Genuss solcher Anerkennung kamen, bestand durch die hohe Arbeitsbelastung in TIRDEP-Projekten die Gefahr, „sich zu vergessen“ (kujisahau) und auf gleichbleibendem Bildungs- und Berufsstatus zu stagnieren; manche Fachkräfte verabschiedeten sich daher bewusst von TIRDEP und gingen „zurück zu den Büchern“, um auf einer höheren Ebene wieder in die Praxis einzusteigen. ${ }^{66}$ Es ist allerdings auffällig, dass zum Zeitpunkt des Interviews (bzw. bis zum Ende ihrer Karriere) viele Angestellte mit vergleichsweise geringerem Bildungsgrad noch den gleichen oder nur einen geringfügig höheren Rang wie zu TIRDEP-Zeiten einnahmen. Bereits damals ranghohen Counterparts hingegen gelang in folgenden Jahren der weitere Aufstieg von den Regionalbehörden und TIRDEP-Projekten in nationale Ministerien, internationale NGOs und globale vernetzte Weltbankprojekte.

\section{Die Aushandlung materieller Anreize}

In mehreren TIRDEP-Projekten war die Transformation materieller Anreize ein Kernstück von Initiativen zur Umgestaltung der Arbeitsbedingungen und Arbeitsdisziplin. Diese Initiativen waren jedoch stets geprägt durch eine sozialräumliche Distanz zwischen expatriates und tansanischem Personal. Tagessätze und in Einzelfällen vereinbarte Gehaltsaufstockungen sollten die Arbeitsmotivation und vor allem ein "full time commitment“ des Projektpersonals aufrechterhalten. ${ }^{67}$ Tatsächlich galt die Zahlung von Taggeldern bald als unverzichtbar, „um einen reibungslosen Ablauf der Aktivitäten zu gewährleisten“, und wenn die tansanischen Behörden Budgetengpässe verkraften mussten und zahlungsunfähig waren, sahen GTZ-ProjektleiterInnen keine andere Alternative, als auch die Sätze der Counterpartleistungen zu übernehmen. ${ }^{68}$ Die Einführung und der graduelle Bedeutungsgewinn von Tages- und Übernachtungsspesen ist einerseits auf deren Normierung durch die tansanische Regierung zurückzuführen - etwa durch

65 Interview \#25, GTZ-Projektleiter.

66 Interview \#53, Tansanischer Landwirtschaftsexperte; Interview \#57, Tansanischer Landwirtschaftsexperte.

67 BMZ, B 213/48211, Project Progress Report No. 1 Rural Development Tanga, Tanga, 30.1.1990. 68 BMZ, B 213/48206, GTZ, Stellungnahme zum Fortschrittsbericht Nr. 24 Gartenbauberatung, 7.11.1989. 
neue Richtlinien für den öffentlichen Dienst. ${ }^{69}$ Andererseits realisierten die ProjektleiterInnen auch schnell, dass Lohnnebenleistungen im Vergleich zur fehlenden Motivation oder Abwesenheit des Personals das kleinere Übel darstellten. Das Szenario war nicht auf Tansania beschränkt: GTZ-MitarbeiterInnen der Zentrale trafen auf ihren Dienstreisen immer wieder auf Personal, das von den staatlichen Trägern schlecht bezahlt wurde, dementsprechend unmotiviert war und andere Einkommensmöglichkeiten suchte. Nicht nur die GTZ, auch andere internationale Organisationen setzten daher auf „Leistungsanreize“, oft in finanzieller Form, „wie eine vornehme Variante der Bestechung“.$^{70}$ Die Genese der per diem-Ökonomie hing auch mit dem Aufstieg partizipativer Methoden und der Intensivierung von Fortbildungen zusammen. Ein guter Teil des Fachpersonals ging kaum noch ins Büro oder ,ins Feld“, sondern „turnte nur noch auf capacity building-Workshops rum“ “ ${ }^{71}$

Zwischen GTZ-ProjektleiterInnen und den Verantwortlichen der Regionalund Distriktbehörden gab es immer wieder Verhandlungen über die Höhe von Tagessätzen, wer einen Anspruch auf Tagessätze habe, in welcher Form die Gelder zu transferieren seien (in bar oder als Gutschein für Güter und Naturalien; direkt an die Begünstigten oder über den Umweg der Regionalverwaltung ausgezahlt) und wer Projektfahrzeuge bereitzustellen habe oder nutzen dürfe. ${ }^{72}$ Die Allokationsfragen waren zudem auch ein häufiger Streitpunkt in den Belegschaften. Ein tansanischer Beamter, der zeitweise die Befugnis hatte $\mathrm{zu}$ entscheiden, welche Fachkräfte im Dienst der Behörde verblieben blieben und welche in geberfinanzierten Projekten eingesetzt wurden, musste mit Beschwerden über unfaire Behandlung umgehen, gerade von jungen KollegInnen, denen damit die Gehaltsaufschläge entgingen. Laut ihm gab es für die „Zurückgebliebenen“ aber zumindest den Ausgleich, bei Dienstreisen die Regierungssätze für Übernachtungen zu bekommen, die drei Mal höher lagen als jene des Projekts. ${ }^{73}$ Um eine gewisse Gerechtigkeit zu wahren, wurde ihnen häufiger die Möglichkeit zu Ausfahrten mit per diem-Entlohnung eingeräumt.

69 Fixe Übernachtungsraten etwa wurden 1976 von der Regierung eingeführt und in den Jahren danach immer wieder angepasst, woran sich TIRDEP zu orientieren hatte. Tanga Library, [untitled folder - TIRDEP Administrative Issues], Circular from Project Coordinator to all TIRDEP Members, Tanga, 3.4.1985; ebd., Circular from Project Coordinator to all TIRDEP Members, Tanga, Februar 1986.

70 Elshorst zit. nach Köhler, Mittler zwischen den Welten, S. 69.

71 Interview \#94, GTZ-Verwaltungsangestellte.

72 Tanga Library, [RCDO], Minutes of a meeting with RCDO, 5.7.1990; ebd., VDP Project Manager an RCDO, Tanga, 12.5.1990.

73 Interview \#55, Tansanischer Beamter. 
Die Gewährung derartiger Zusatzleistungen blieb immer durch ein entwicklungspolitisch begründetes Unbehagen gekennzeichnet. In der Perspektive von GTZ-ProjektleiterInnen waren materielle Anreize immer eine Gratwanderung zwischen kurzfristiger Notwendigkeit und „nachhaltigen“ Lösungen. ${ }^{74}$ Eine Projektleiterin etwa berichtete der DSE von einem Bildungsworkshop (finanziert von der DSE selbst), sie sei von den „außerordentlich üppigen Tagegelder[n]“ überrascht gewesen, die „etwa das Fünffache von dem [ausmachten], was bei solchen Gelegenheiten in Tansania gezahlt“ wurde und „etwa einem halben Monatsgehalt“ entsprach. Der tansanische Workshopleiter habe versucht, ihr „Spesen für 7 Tage aufzudrängen und reagierte erstaunt, daß ich nur für die effektiven Reisetage annehmen wollte, er meinte, man habe ja so viel Geld und müsse es ausgeben“. Schließlich kam sie zu ihren Bedenken, was die mittel- und langfristige Perspektive anging:

Natürlich gönne ich den Tanzaniern Spesen, die Ihnen hohe Ersparnisse ermöglichen, im Gegensatz zu ihren Gehältern. Jedoch sehe ich die Gefahr, daß Teilnehmer solcher aus dem Ausland finanzierter Workshops nicht mehr bereit sein werden, für ihre Ministerien und Projekte im Land zu den üblichen Spesensätzen zu arbeiten. ${ }^{75}$

Ihre Machtposition und die soziale Distanz zu denjenigen, die kurz- und mittelfristig auf die Tagessätze angewiesen waren, erlaubte expatriates, auf abstrakte entwicklungspolitische Prinzipien und Langfristigkeit zu pochen. Damit rationalisierten sie auch, die ungenügenden lokalen Gehälter anstelle der im Vergleich überdimensionierten expatriate-Gehälter als Maßstab anzusetzen. Die konkrete Höhe des GTZ-Gehalts wurde vor TansanierInnen im Übrigen zwar immer geheim gehalten, die Dimensionsunterschiede waren aber schon allein durch den anderen Lebensstil offensichtlich. Argumente der Nachhaltigkeit und Gleichheit wurden auch in TIRDEP-Teamsitzungen bemüht, um festzuhalten, dass eine direkte Anstellung qualifizierter Counterparts -analog zu expatriates, die lokale statt GTZ-Verträge hatten und ihr Gehalt in Devisen erhielten - nicht opportun war: Das würde eine Klassenstruktur im Gehaltsschema etablieren und die Integrationsziele von TIRDEP ad absurdum führen. ${ }^{76}$ Das Argument der Gleichheit bezog sich immer auf die tansanischen Vergleichsgrößen, nie auf die

74 Tanga Library, [General Correspondences], VWSP Project Manager to TIRDEP Project Managers and Coordination Unit, Tanga, 19.9.1989.

75 Privatarchiv S. R., [Adult Education Corr. Min. Ed. DSE etc.], PESP Project Manager S. R. an DSE, Tanga, 25.6.1987.

76 Privatarchiv S. R., [Team-Meeting Minutes \& Papers 1985-89, Bd. 1], Minutes of TIRDEP team meeting, o.0.1.12.1986. 
Unterschiede zwischen expatriates und tansanischen Counterparts. Das GTZPersonal stand selbst an der Spitze einer (real existierenden) sozioökonomischen Hierarchie und argumentierte, die (hypothetische) Herausbildung einer Klassenstruktur verhindern zu wollen.

Das deutsche ${ }^{77}$ TIRDEP-Entwicklungspersonal hegte, von Ausnahmen abgesehen, eine generelle Sympathie für die Ziele von Ujamaa und gründete die eigene Tätigkeit auf zwei entwicklungspolitischen Prinzipien: Erstens sollten die Interventionen an den Bedürfnissen der „Zielgruppen“ ausgerichtet sein; zweitens sollte die tansanische Seite in die Entscheidungsprozesse einbezogen werden. ${ }^{78}$ Anfangs noch enthusiastisch vertretene entwicklungspolitische Ideale mochten nach einiger Zeit vom branchenüblichen Firnis aus Zynismus überzogen werden, aber sie blieben Teil der Grundhaltung. Tanga bildete für sie ein Wirkungs- und Experimentierfeld und ermöglichte, sich in der entwicklungspolitischen Avantgarde zu verorten. Die Wirksamkeitsfantasien waren dabei nicht völlig aus der Luft gegriffen, sondern durchaus in Erfahrungen und realen Einflussmöglichkeiten begründet. Selbst „vier Greenhorns“, die „quasi frisch von der Uni“ kamen, konnten in der Erinnerung eines ehemaligen GTZ-Experten den ganzen Distrikt Lushoto mit einem Landwirtschaftsprojekt in ihr „Labor für Social Engineering“ umwandeln. ${ }^{79}$ Die Wirksamkeitsfantasien gingen dabei oft über den „eigenen“ geografischen Bereich hinaus. Projektbesuche und Absichtsbekundungen von Nyerere und Ministern etwa weckten bei GTZ-ExpertInnen Hoffnungen, dass Ansätze im eigenen Projekt als modellhaft anerkannt und landesweit übernommen würden. Viele ProjektleiterInnen genossen und nutzten den großen Einfluss in ihren Projekten und reagierten empfindlich, wenn andere ExpertInnen in ihr „Hoheitsgebiet“ eindringen wollten. ${ }^{80}$

Retrospektiv wurden die in Archiven und Interviews bisweilen aufscheinenden Konflikte einem gemeinsamen Korpsgeist der „Tirdeppen“, wie sich die Deutschen im Programm selber nannten, untergeordnet: „Wir haben alle in Tanga

77 Nicht alle der GTZ-ExpertInnen (bzw. auch expatriates mit Lokalverträgen) in TIRDEP waren Westdeutsche - es waren auch Briten, Niederländer, ein Tscheche, ein Äthiopier, eine Bulgarin, eine DDR-Bürgerin und andere Nationalitäten darunter. Angesichts der Bindung an die GTZ wird im Folgenden der Einfachheit halber trotzdem von „deutschem Personal“ gesprochen, zumal dies auch weitgehend mit den Wahrnehmungen übereinstimmt, die in den Interviews ausgedrückt wurden. Zusätzlich zum GTZ-Personal arbeiteten auch Freiwillige vom DED sowie VolontärInnen aus Schweden, den Niederlanden und Japan in TIRDEP-Projekten.

78 Privatarchiv S. R., [BMZ-Evaluierung 1991 + TIRDEP Selbstevaluierung], TIRDEP Coordinator an Members of Coordination Group, o.O., 5.4.1984.

79 Interview \#26, GTZ-Experte.

80 Interview \#94, GTZ-Projektleiter. 
an dem gleichen Strumpf gestrickt.“81 Dieses „wir“ bezog sich aber in der Regel nur auf das entsandte GTZ-Personal-Personal. Rückblickend waren manche verwundert darüber, dass sie als „Weißnasen“ an einem Tisch in Tanga solche Entscheidungsmacht in der Region besessen hatten: Die TIRDEP-Koordinationseinheit sei „quasi eine Parallelregierung“ gewesen, „völlig absurd aus heutiger Sicht betrachtet. Da saßen lauter Weiße um den Tisch rum und haben überlegt, was sie in Tanga machen. [...] Da war niemals ein Tansanier. “82 Dabei erinnerten sich viele auch an den experimentellen Charakter der eigenen Tätigkeit, der wenig mit einer technischen Anwendung von Expertise, sondern einem ständigen kreativen Suchen nach Lösungsansätzen zu tun hatte: „Man hat uns im Prinzip fünf Jahr bosseln lassen. Wir haben fünf Jahre probiert." ${ }^{83}$ Tanga galt, so ein ehemaliger tansanischer Counterpart, weithin als „German territory“, weswegen auch das Interesse anderer Geber an der Region in den späten 1980er-Jahren misstrauisch als mögliche Konkurrenz oder Einmischung beäugt wurde. ${ }^{84}$ Bis zu diesem Zeitpunkt gehörten GTZ-Entsandte hohen Ranges zu den Akteuren, die Ressourcenflüsse in der Region entscheidend mitsteuerten. Die Beziehungen zwischen expatriates und tansanischen Counterparts gestalteten sich trotz dieses klaren Machtgefälles in konkreten Fällen jedoch oft komplexer.

\subsection{Die asymmetrische Interdependenz der Counterpartbeziehungen}

Wer in TIRDEP als (tansanischer) Counterpart galt, wurde nie verbindlich festgelegt; die Verwendung des Begriffs „Counterpart“ war nicht einmal zwischen den verschiedenen TIRDEP-Projekten einheitlich geregelt. In einer Projektübersicht aus dem Jahr 1988 scheinen einige Projekte auf, für die nur ein Counterpart angeführt wird, in einem anderen hingegen 59 - während es jeweils nur ein bis fünf GTZ-ExpertInnen pro Projekt waren. Aus der Vielfalt der Projekte und Ansätze ergibt sich zwangsläufig, dass die Counterpartbeziehungen äußerst heterogen ausfielen. Unabhängig vom Status der Beteiligten glichen sie sich aber darin, dass sie durch eine asymmetrische Interdependenz geprägt waren. Die Interdependenz gründete auf den höheren Ebenen darin, dass die höherrangigen Beteiligten aller

81 Interview \#26, GTZ-Experte.

82 Ebd. Diese Äußerung ist deutlich zugespitzt, Protokolle der Sitzungen zeigen, dass TansanierInnen durchaus an Treffen teilnahmen - allerdings durchaus nicht immer und wenn, dann üblicherweise in geringer Zahl.

$83 \mathrm{Ebd}$.

84 Interview \#52, Tansanischer Landwirtschaftsexperte; Interview \#89, Schwedischer Volontär. 
Seiten meist ein handfestes materielles und karrierebedingtes Interesse am „Projekt“ und seiner Fortführung haben. Wie die Regierungsberater (Kapitel 6) war das GTZ-Projektpersonal auf strategische Allianzen in Tanga und - zur Übertragung von Konzepten auf die nationale Ebene - in Dar es Salaam und Dodoma angewiesen, wenn die Interventionen keine isolierten Insellösungen bleiben sollten. ${ }^{85}$ Auf mittleren und unteren Ebenen wiederum waren die expatriates von Vermittlungsfähigkeiten und Sprachkenntnissen ihrer Counterparts abhängig, da ohne sie Aktivitäten nicht umgesetzt werden konnten. Die Asymmetrie in dieser Interdependenz ergab sich aus der ungleichen Ressourcenverteilung und den Rollenverständnissen. Dem GTZ-Personal stand ökonomisches und institutionelles Kapital zur Verfügung, das auch die entsprechenden Machtvorteile sicherte. Die Basis dieses Kapitals lag allerdings oft extern im Ausland bzw. in ihrer Vermittlerposition zwischen lokaler und internationaler Ebene. AkteurInnen auf tansanischer Seite hingegen konnten ihr lokal wirksames politisches und soziales Kapital einsetzen und versuchten, ihre Position im Inneren durch Extraversion zu stärken.

Die Feststellungen zu den widersprüchlichen Rollenerwartungen an die EntwicklungsexpertInnen (Kapitel 5.4) gelten auch für TIRDEP, insbesondere was die Entscheidung zwischen Beratung oder Ausführung anbelangt. Der Fokus liegt im Folgenden auf drei Rollen in den Counterpartverhältnissen, die allesamt verdeutlichen, dass Projekte wie jene von TIRDEP immer einen umkämpften $\mathrm{Zu}$ gangspunkt zu Ressourcen darstellen. Beteiligte auf den obersten Ebenen übten als (1) gatekeeper Einfluss auf die Ressourcenflüsse aus. Zur Aufrechterhaltung der Ressourcenflüsse gab es gegenseitige Gefälligkeiten, die den inoffiziellen Spielregeln, nicht aber dem offiziellen entwicklungspolitischen Skript entsprachen. Die Repräsentation der Projektrealitäten im offiziellen Skript war aber eine ebensowichtige Funktion. Tansanische Counterparts und GTZ-ProjektleiterInnen fungierten daher gleichermaßen als (2) EntwicklungsmaklerInnen, die zwischen den verschiedenen Interessen und Umfeldern vermittelten und Ressourcenflüsse durch die Aneignung bestimmter Diskurse sicherzustellen suchten. Drittens ging es vor allem auf der Ebene des ausführenden Personals um eine „Inwertsetzung“: Hier sahen sich expatriates als (3) InvestorInnen. Anhand dieser drei Rollen wird die gegenseitige, aber asymmetrische Abhängigkeit in den Counterpartverhältnissen der Entwicklungsarbeit deutlich.

85 Tanga Library, [PFK '92 -Decentraliz, PRDVL, Reports], o.A., Discussion paper No. 1 (Second Draft), 28.1.1992. 


\section{Gatekeeper: Die Steuerung der Ressourcenflüsse}

Die einflussreichsten Counterparts lassen sich im Anschluss an Frederick Cooper als gatekeeper $\mathrm{zu}$ beschreiben. Wie Cooper argumentiert, war die wichtigste Funktion des postkolonialen Staates - geerbt vom Kolonialregime - die Kontrolle der Schnittstelle zwischen nationaler Wirtschaft und Weltmarkt. ${ }^{86}$ Mit der Dezentralisierung in Tansania hatten die Regional Commissioner (Parteifunktionäre mit Ministerrang) eine derartige Torwächterfunktion für die Region inne. Pius Msekwa - der schon als Universitätsrektor der DDR Sorgenfalten auf die Stirn getrieben, aber der BRD einen guten Empfang bereitet hatte (siehe Kapitel 7) wurde von Nyerere auf diese Funktion bestellt, um Japan den Import von Traktoren zu ermöglichen, was sein Vorgänger noch behindert hatte:

So one of your responsibilities is go and make sure the Japanese can deliver without harassment. I know that for sure because when Mwalimu Nyerere transferred me from Tabora to Kilimanjaro, he told me that the Japanese are being frustrated in Kilimanjaro. [...] So [Nyerere] said [to me], make sure the bureaucracy does not frustrate the Japanese. [...] If it's a small question of issuing a license, what's the problem there? They want to import a tractor, what's the problem ${ }^{87}$

Laut Msekwa war es für Regional Commissioner eine Kernkompetenz, bei Ausschreibungen für Ausrüstungslieferungen und Investitionen Legalität einerseits und hinter geschlossenen Türen bereits getroffene Entscheidungen andererseits in Einklang zu bringen (,How to favor somebody without breaking the law“) also die Harmonisierung von offiziellem Skript und inoffiziellen Aushandlungsergebnissen. ${ }^{88}$ Auch TIRDEP war auf Importe und damit Importlizenzen angewiesen. Außerdem waren die Regionalbehörden essenziell, um die Arbeitserlaubnis auszustellen und zu verlängern oder die Bereitstellung von Unterkünften zu beschleunigen, Personal- und Projektvorschläge $\mathrm{zu}$ akzeptieren und finanzielle, personelle und materielle Ressourcen („Counterpartleistungen“) bereitzustellen. Die gatekeeper auf der tansanischen Seite hatten in vielen Fragen eine faktische Vetomacht und gaben dem damaligen GTZ-Projektkoordinator das Gefühl, Gegenleistungen bereitstellen $\mathrm{zu}$ müssen:

Das prinzipielle Verhältnis durfte man schon nicht stören. Weil Macht ist ja informell anders verteilt als man glaubt. Die können einem ja sonstwas- Und es wird ja doch sehr viel, na ja, nicht ganz in der Legalität gemacht. Weil man kann ja überall eine Illegalität rausleiten, bei

86 Cooper, Africa since 1940, Kap. 7.

87 Interview mit Pius Msekwa, Dar es Salaam, 23.11.2014.

88 Ebd. 
jeder Sendung die reinkommt, was also zollpflichtig ist, es gibt ja unendliche Bestellungen. Da sind die immer recht großzügig gewesen. Aber da musste man auch entsprechend sein. ${ }^{89}$

Selbst PolitikerInnen auf Distrikt- und Dorfebene konnten als gatekeeper Einfluss ausüben. Ein tansanischer Beamter der Regionalbehörde mit viel Felderfahrung meinte: ,[I]f you go into villages without involving those party leaders there, you are in hot soup. They can block you. “90 Auf Dorfebene waren die Parteiautoritäten insbesondere notwendig, um die Bevölkerung zu mobilisieren. Ein District Comissioner, laut einem ehemaligen Projektleiter ein „Parteihengst“, verbot einem deutschen TIRDEP-Gast die Einreise „obwohl es [dafür] gar kein Gesetz gab“ und der Besucher ein gültiges Visum hatte. Der ehemalige TIRDEP-Koordinator deutete dies als Machtdemonstration und „eine Art Disziplinierung. “91 Die Handlungsoption der Evasion, also „lästigen“ Regierungscounterparts und „Parteisoldaten“ möglichst aus dem Weg zu gehen, barg das Risiko, sie durch Missachtung ihrer Autorität oder Expertise gegen sich aufzubringen. ${ }^{92}$ Da das GTZPersonal persönlich (Arbeitserlaubnis, Unterkunft) wie professionell (Umsetzung von Projektaktivitäten) von den gatekeepern abhängig war, schien es vielen ratsamer, sie zu hofieren. So gab es gelegentliche soziale Veranstaltungen und Empfänge, zu denen die wichtigsten Counterparts, bisweilen aber auch weitere politische und administrative gatekeeper eingeladen wurden - etwa der als „harter Knochen“ wahrgenommene Beamte, der in der Tanga-Region für die Ausstellung und Verlängerung der Arbeitserlaubnis zuständig war. ${ }^{93}$ Mit Glück und geschicktem Umgang konnten bei solchen Anlässen beidseitig produktive Beziehungen entstehen. Die Freundschaft zwischen der Ehefrau des Regional Commissioners Kingunge Ngombale-Mwiru und der Gattin eines Projektleiters etwa habe zu einer Verringerung des gegenseitigen Misstrauens geführt. ${ }^{94}$ Gezielt wurden die wichtigsten Köpfe der Region auch für Arbeitsaufenthalte in die BRD eingeladen, um Unstimmigkeiten über Richtlinien und Ressourcenallokationen in Bonn oder Eschborn auszubügeln. ${ }^{95}$ GTZ-ExpertInnen (ebenso wie hochrangige tansanische Beamte) waren auch gatekeeper in dem Sinne, dass sie Counterparts

89 Interview \#25, GTZ-Projektleiter.

90 Interview \#80, Tansanischer Landwirtschaftsexperte.

91 Interview \#25, GTZ-Projektleiter.

92 Interview \#26, GTZ-Experte.

93 Interview \#26, GTZ-Projektleiter; Interview \#80, Tansanischer Landwirtschaftsexperte.

94 Interview \#27, GTZ-Projektleiter. Wie in Kapitel 3 erwähnt zählte Ngombale-Mwiru zum linken, marxistischen Flügel der Partei.

95 PAAA, ZW 116 859, BRD-Botschaft an AA, Dar es Salaam, 29.8.1977; BArch Koblenz, B 213/ 33081, Sartorius/Zils (GTZ) an BMZ, Eschborn, 19.11.1979. 
und Projektpersonal für Auslandsstudium und Fortbildungen vorschlugen, also buchstäblich Türen ins Ausland und damit zu neuen Möglichkeiten der Kapitalakkumulation zu öffnen vermochten - oder diese geschlossen halten konnten.

Die Beziehungsarbeit führte bisweilen dazu, dass Regelungen zur Ressourcenallokation im Falle von Transportmitteln und anderen Investitionen gedehnt und umgangen wurden. Selbst die höchsten Behörden in der Region wurden von der Wirtschaftskrise und Ressourcenknappheit erfasst, privat wie beruflich, wie sich ein ehemaliger Projektkoordinator erinnerte, manche Counterparts galten ihm als „arme Hunde“ und „selbst der Regional [Development] Director“, der staatliche Verwaltungschef der Region, „war zeitweise ohne Auto“ gewesen, wie er sich erinnerte. ${ }^{96}$ Die Aneignungsstrategien der tansanischen Behördenleiter können aber nicht einfach als Selbstbereicherung gelesen und auf egoistische Beweggründe reduziert werden; manche der leitenden Beamten genossen bei GTZ-ExpertInnen den Ruf, „immer korrekt“ gehandelt zu haben. ${ }^{97}$ Wie Leander Schneider gezeigt hat, sind rein materialistische Theorien unzureichend, um die Handlungsweisen von Partei- und Verwaltungskräften im Tansania der 1970er Jahre zu verstehen, da ihre Interessen stark von den institutionellen und diskursiven Gegebenheiten abhingen, in denen sie operierten. ${ }^{98}$

Eine Haupttätigkeit vieler hoher tansanischer Counterparts war ein „Scouting nach Ressourcen“, das keinesfalls ausschließlich, aber doch zu einem wichtigen Teil nach außen orientiert war (Abbildung 8.1). ${ }^{99}$ Die Leiter der Regionalbehörden erhofften sich die Unterstützung ihrer offiziellen Stellen mit Fahrzeugen, Fortbildungen und Finanzen. Hier kann nicht einzig von Gemeinplätzen wie Korruption und Neopatrimonialismus ausgegangen werden, auch der politische Druck, Ergebnisse zu liefern - meist von oben, bisweilen auch von unten - ist in Betracht zu ziehen. TIRDEP-eigene Lastwagen wurden z.B. für die finale, von Zwangsmaßnahmen geprägte Phase der Umsiedlungsaktionen „zweckentfremdet“. ${ }^{100}$ Ihre Requirierung im Zuge des Uganda-Krieges für militärische Zwecke wurde vom Projektkoordinator 1979/80 verhindert, aber TIRDEP-Fahrzeuge

96 Interview \#25, GTZ-Projektleiter; ähnlich Interview \#26, GTZ-Experte.

97 Interview \#49, GTZ-Projektleiterin.

98 Schneider, Government of Development, S. 129.

99 Interview \#84, Tansanischer Entwicklungsplaner und Counterpart; Interview \#55, Tansanischer Beamter. Der Entwicklungsplaner der Region, der gleichzeitig Counterpart für den GTZKoordinator war, musste auch alljährlich bei der Zentralregierung um die Bereitstellung von Mitteln für Tanga kämpfen: jede Region wollte ein möglichst großes Stück des „Budgetkuchens“ für sich.

100 Interview \#102, GTZ-Experte. In politisch heiklen Fällen wie diesem ist dann allerdings auch die bundesdeutsche Botschaft eingeschaltet worden. 
wurden in anderen Fällen genutzt, um Prüfungsunterlagen in entlegene Schulen zu transportieren. Damit erfüllte die Regionalverwaltung die ihr gestellten Aufgaben und überwand mit Hilfe der externen Ressourcen lokale Engpässe. Die kurzfristigen Ergebnisse dieser Extraversionsstrategien konnten materielle Strukuren und soziale Dienstleistungen (wie die rechtzeitige und ordnungsgemäßge Durchführung von Schulprüfungen) umfassen, aber auch nationale Zielstellungen.

In den Worten eines GTZ-Projektkoordinators waren die gatekeeper ihrer politischen Aufgabe nach vor allem „Devisenanschaffer“ ${ }^{101}$, die zur Schließung des tansanischen Haushaltsdefizits beitragen sollten - selbst wenn die Gelder immer an konkrete Maßnahme gebunden waren. ${ }^{102}$ Die politische Aufgabe der

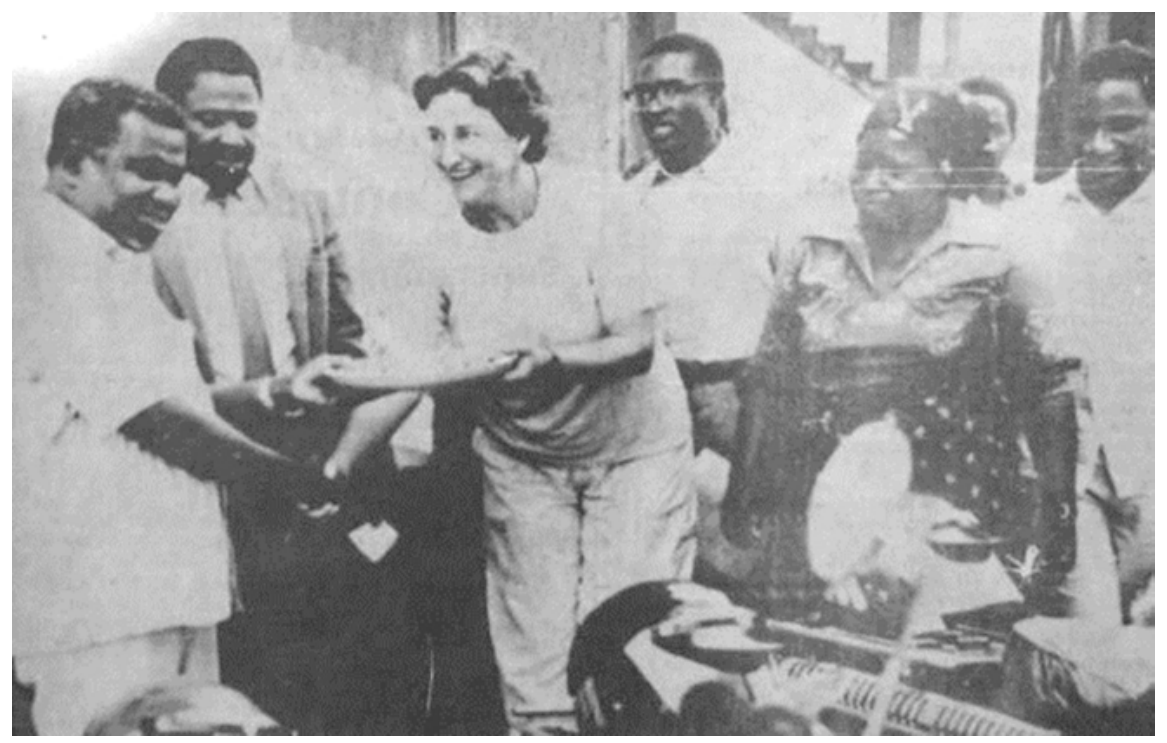

Abbildung 8.1: Ressourcentransfer als Win-win-Situation: Regional Development Director Idarous Abubakar erhält von der GTZ-Leiterin des Schullandwirtschaftsprojekts PESP die Schlüssel zu den zwölf Motorrädern, die das Projekt an die Region übergibt. Die Motorräder sollten den Schulinspektoren ermöglichen, die Umsetzung des Projektes an 644 Schulen in der Region effektiver voranzutreiben. (Quelle: Daily News, 10.6.1986)

101 Sogar in einem BMZ-Bericht wurde dieser - keinesfalls entwicklungspolitische - Beitrag geschätzt. TIRDEP entlastete vor allem durch die vielen Importe, z.B. von Medikamenten für veterinärmedizinische Zwecke, den Devisenhaushalt. Tanga Library, BMZ, Main Report on the inspection of the Technical Cooperation Project TIRDEP, Bonn, 1.12.1982, S. 75.

102 Interview \#25, GTZ-Projektleiter. 
„Devisenbeschaffung“ war freilich in den 1980er-Jahren dringlicher als noch zuvor, was einen klassischen Reibungspunkt in den Counterpartbeziehungen noch prononcierte. Da infolge der Überbewertung des tansanischen Schillings in bestimmten Jahren die Importe zeitweise scheinbar günstig waren, befürworteten viele tansanische Counterparts kapitalintensive, moderne Technologien in der Erwartung, dass die Geber bei größeren Investitionen auch bereitwilliger die Instandhaltungskosten und Beschaffung von Ersatzteilen übernehmen würden. Hauptsächlich aber argumentierten sie für die Mechanisierung des Feldbaus durch Traktoren (statt seiner „Bestialisierung“ durch Ochsen) oder die Installation großflächiger, komplexer Rohrleitungsanlagen (anstelle von leicht auf Dorfebene zu wartenden Flachbrunnen) aufgrund einer Disposition für möglichst „moderne“ Technologien, die mit Fortschrittsvorstellungen im Einklang standen. ${ }^{103}$ Auf BRD-Seite hatte man noch in den 1970er-Jahren, z. B. im Schulbau, auf „deutsche Standards“ gesetzt, sodass in der Technologiefrage Einstimmigkeit bestanden hatte. In den 1980er-Jahren, als die breite Kritik an „weißen Elefanten“ und Entwicklungshilferuinen angesichts zahlreicher gescheiterter Projekte ihre Wirkung entfaltete und der Glaube an eine nachholende industrialisierende Entwicklung versiegte, bevorzugten in Tanga eingesetzte GTZ-ExpertInnen zunehmend angepasste Technologien und arbeitsintensive Selbsthilfekonzepte.

Wie aber schon im obigen Zitat des GTZ-Projektleiters deutlich wird, war der Ausgang von Diskussionen durch das wirtschaftliche Ungleichgewicht weitgehend vorbestimmt, wie auch ein tansanischer Counterpart meinte:

The structure as such, the [Tanzanian] government had the final say. But when you relate to resources, it's the Germans who are having the final say. They say okay, you want to build a school. But you don't have money for the school. We have money for livestock development. What do you do? [...] [The] government office has power as far as decision-making is concerned. But when you come to resources you have to somehow agree with what the donors say. ${ }^{104}$

103 Tanga Library, BMZ, Main Report on the inspection of the Technical Cooperation Project TIRDEP, Bonn, 1.12.1982, S. 58, 73; Interview \#90, Tansanischer Ingenieur und Counterpart; Privatarchiv S. R., [PESP Kilimo Handouts + Dev. Articles], Andreas Bittner, Gespräche mit der Praxis, Auswertung von 35 Interviews mit unter Vertrag stehenden deutschen Fachkräften in der Entwicklungszusammenarbeit, Wiesbaden, Mai 1984, S. 8.

104 Interview \#7, Tansanischer Entwicklungsplaner und Counterpart. Juma Ngasongwa kommt im Rahmen seiner Studie über RIDEPs in Tansania zu dem Schluss, dass der tansanische Counterpart zum GTZ-Projektkoordinator in Tanga sich durch Machtlosigkeit in den alltäglichen Entscheidungsprozessen auszeichnete, zumal er auch seinen physischen Sitz nicht im TIRDEPBüro hatte. Ngasongwa, Evaluation, S. 300. 
Dementsprechend blieb es in den meisten Fällen bei Ochsen und Flachbrunnen. Während tansanische Beamte in Interviews, die Anfang der 1980er-Jahre in Tanga geführt wurden, bemängelten, dass „Entscheidungen der deutschen Projektmanager [...] ihnen lediglich zur Kenntnisnahme vorgelegt“" wurden und klagten, „kaum Möglichkeiten [zu] haben, auf die Projektausführung Einfluss zu nehmen“105, findet sich in späteren Interviews eine Resignation im Umgang mit der Abhängigkeitssituation und ihrer ungleichen Machtverteilung: Es handele sich nun einmal um „das Geld des Gebers“ (hela ni zake). ${ }^{106}$ Lars Johannsson, ein Schwede, der selbst als Volontär und Sachverständiger für TIRDEP tätig war, konstatierte in einer Publikation von 2001, dass die deutsche Entscheidungshoheit in Budgetfragen nicht hinterfragt wurde, weil sie die Verfügbarkeit von Ressourcen sicherstellte: „Tanzanian project staff were generally in support of this arrangement because they found the demand for donor accountability reasonable, but also because the arrangement allowed the project to provide them with decent work facilities (e.g. transport, training) compared to colleagues in the regular extension service." ${ }^{\text {"107 }}$

Wenn in (rezenten) Interviews Kritik an der BRD-Entwicklungshilfe und deutschen ProjektleiterInnen geübt wurde, dann weil diese - als gatekeeper im Reich „ihres“ jeweiligen Projekts agierend und immer darauf bedacht, die Projektressourcen nicht in anderen Kanälen versickern zu lassen - sich nicht zur Allokation von Mitteln überreden ließen, wie es die Counterparts wünschten. Schon in den 1980er Jahren verwiesen Counterparts auf andere TIRDEP-Projekte, die diese Inputs leisteten, um die bundesdeutschen Akteure zu überzeugen. ${ }^{108}$ Ein tansanischer leitender Beamter, der in der DDR studiert hatte, erinnerte sich an einen Konflikt mit einem GTZ-Experten, in dem es um den dringenden Kauf von Medikamenten gegen eine plötzlich aufgetretene Viehkrankheit ging. Dabei leitete er aus dem Besitz von Ressourcen auch soziale Verantwortung ab: „Wir haben uns gestritten, ich habe gesagt, ,Wenn sie [die Rinder] sterben ist das Ihre Schuld, denn die Regierung hat kein Geld.““109 Diese Argumentationsstrategie sei erfolgreich gewesen, er habe das Geld bekommen, aber sich dann mit den hartnäckigen Rückzahlungsforderungen des GTZ-Experten auseinandersetzen müssen. Der restriktive Umgang von GTZ-Projektverantwortlichen mit Finanzen wiederum

105 Wirth, Aspekte, S. 152-153.

106 Interview \#58, Tansanische Gartenbauexpertin; ähnlich Interview mit Pius Msekwa, Dar es Salaam, 23.11.2014.

107 Johansson, Ten Million Trees Later, S. 154.

108 Tanga Library, [General Correspondences], VDP Project Manager an RCDO, 13.11.1985; ebd., [RCDO], Minutes of a meeting with RCDO, 5.7.1990.

109 Interview \#78, Tansanischer Landwirtschaftsexperte. 
erklärt sich auch daraus, dass sie für die korrekte Verwendung der Mittel bürgen mussten und in Fällen fehlender Belege privat zu haften hatten. ${ }^{110}$ Während sowohl auf deutscher wie auch tansanischer Seite gatekeeper zu finden waren, so entschieden sie doch über den Zugang zu verschiedenen Typen von Ressourcen.

\section{Entwicklungsmakler: Übersetzung und Interessenvermittlung}

Verflochten mit der Funktion des gatekeepers war die des Entwicklungsmaklers; beide Funktionen waren auch in Personalunion anzutreffen. Voraussetzung für eine Maklerrolle war genau wie im Falle des gatekeepers die Positionierung an einer wichtigen Schnittstelle zwischen „innen“ und „außen“ oder zwischen verschiedenen Institutionen. Die Position erlaubte es, externe Ressourcen zu mobilisieren. Dafür mussten Diskurse in einer Art verwendet werden (z.B. in der Projektbeschreibung), dass sie für alle involvierten Seiten - insbesondere aber die Geberseite - akzeptabel erschienen. Für die Entwicklungsmaklerposition war (und ist) kennzeichnend, dass hier Diskurse und Handlungen für andere Akteure übersetzt werden. Die Personen, die diese Rolle ausfüllten, konnten ihre eigene Position so weiter stärken. ${ }^{111}$ Am deutlichsten werden diese Aspekte am Beispiel der beiden höchsten Counterparts in TIRDEP, dem GTZ-Programmkoordinator einerseits und dem tansanischen Entwicklungsplaner der Region andererseits. Ein ehemaliger GTZ-Programmkoordinator beschrieb seine Mittlerrolle im Interview als Übersetzung zwischen verschiedenen Kulturen, wobei er die kulturelle Differenz auch als Legitimationsgrund angab, Berichte und Anträge ohne Beteiligung der tansanischen Counterparts geschrieben zu haben:

Und wenn überhaupt eine Änderung stattfinden konnte, konnte ich sie bewirken, bei der Regierungsdelegation, denn ich bin der einzige der sagen konnte, „Nee, lieber Herr Ministerrat, das ist ja nischt, kann man da nicht was Anderes machen.“ Aber dazu waren die [Tansanier] nicht in der Lage. [...] Alle Papiere, die nach Deutschland gehen, habe ich geschrieben. Und [den tansanischen Counterpart], den konnte man so nicht allein lassen. Der hat ja gar keine Ahnung, wie man mit der europäischen Bürokratie umgehen kann, mit der

110 So in einem Fall, als Projektmittel entwendet wurden, aber den beschuldigten (tansanischen) Buchhaltern keine Schuld nachgewiesen werden konnte. Interview \#49, GTZ-Projektleiterin; vgl. auch Interview \#8, GTZ-Projektleiterin.

111 Diese Merkmale entsprechen dem Konzept vom development broker, auch wenn dieses in der Literatur eher für Akteure aus der Zivilgesellschaft angewendet wird, die in der Mittlerposition zwischen aid business und lokaler Bevölkerung stehen. Siehe z. B. Thomas Bierschenk u. a., Local Development Brokers in Africa. The Rise of a New Social Category. Arbeitspapier Nr. 13, Mainz 2002. 
Ministerialbürokratie. [...] Und da war ich natürlich schon wichtig als mode[rierender], als verbindender Mann. Ich hab’s natürlich den Tansaniern möglichst recht machen wollen, nicht. Und als Früherer vom Ministerium hatte ich natürlich ein besseres Kenntnis oder Gespür was möglich ist und was nicht möglich ist. ${ }^{112}$

Auffällig ist, dass der ehemalige Projektkoordinator sich hier aufgrund seines kulturellen Kapitals - insbesondere seiner Erfahrungen im Umgang mit der bundesdeutschen Bürokratie und seinem zum „Gespür“ gewordenen Verständnis für Handlungsräume innerhalb des „Haushaltsprozesses“ und Verwaltungsapparats - eine Ausnahmeposition an der Schnittstelle zusprach. Wie er schrieben sich auch andere Koordinatoren und ProjektleiterInnen zu, gegenüber den deutschen Stellen vor allem die Interessen der Counterparts bzw. der tansanischen Zielbevölkerung vertreten zu haben, was wiederholt zu Konflikten mit der GTZZentrale oder dem BMZ geführt habe. Archivakten stützen diese Darstellung in einigen Fällen. Die immer wieder aufflammenden „Grundsatzdiskussionen“ über Ujamaa und fehlende „Leistungsanreize“ für die ländliche Bevölkerung in der GTZ und im BMZ etwa stellten das gesamte TIRDEP-Programm immer wieder infrage; die Projektkoordinatoren positionierten sich hier tatsächlich meist als Befürworter der tansanischen Entwicklungspolitik. ${ }^{113}$

GTZ-ProjektleiterInnen hatten auch die Aufgabe, Projektrealitäten zu vermitteln und die Repräsentationen an aktuelle diskursive Trends und politische Sensibilitäten anzupassen, um Beziehungen und Ressourcenflüsse aufrecht zu erhalten. ${ }^{114}$ Diese Übersetzungs- und Überzeugungsfunktion war besonders dann gefragt, wenn die lokalen Projektrealitäten an die „unendlich vielen Besucher [...] verkauft" werden mussten, die die Projekte vor Ort begutachteten. Hochrangige BeamtInnen und PolitikerInnen, die Presse, BMZ-Delegationen oder Evaluationsteams gehörten zu den Gästen und konnten mit ihren Meinungen und Interventionen den weiteren Projektverlauf empfindlich beeinflussen. ${ }^{115}$ Dass etwa im Rahmen eines „Selbsthilfe“-Projektes, das auf der Mobilisierung lokaler Arbeitskraft basieren sollte, dann plötzlich mehrere Lastwagen auffuhren, um einen

112 Interview \#25, GTZ-Projektleiter.

113 Siehe z.B. BArch Koblenz, B 213/33065, Hildenbrand (GTZ), Arbeitspapier: Beurteilung der politischen Situation in Tansania und ihre Auswirkungen auf die durch die BRD geförderten Projekte, o.O., 27.1.1977; sowie die entsprechenden Reaktionen auf dieses Arbeitspapier in der gleichen Akte, v. a. ebd., TIRDEP-Projektkoordinator an die Mitarbeiter von TIRDEP, Hannover, 9.5. 1977.

114 Mosse, Cultivating Development; Rottenburg, Weit hergeholte Fakten.

115 Siehe z. B. Tanga Library, TIR 1/13, TIRDEP Project Progress Report: Support to the Regional Development Director in the Regional Commissioner's Office, July-December 1982, Januar 1983, S. 14. Das Zitat stammt aus Interview \#25, GTZ-Projektleiter. 
Straßenabschnitt rechtzeitig vor einem Ministerbesuch im Jahr 1988 fertigzustellen, ${ }^{116}$ gehörte zu den üblichen Repräsentationsstrategien, mit denen die Mächtigen nicht erst seit Potemkin getäuscht werden. Bundesminister Klein jedenfalls ließ sich überzeugen (oder spielte das Spiel mit, ohne sich etwas anmerken zu lassen), lobte in einem anschließenden Interview, dass die Straße von den „Einwohnern selbst identifiziert und gewünscht [...] und selbst gebaut worden“ war und sprach sich bei aller Kritik an TIRDEP für die Fortführung dieses konkreten Projekts aus. ${ }^{117}$ In der Entwicklungsarbeit sind solche Strategien, in denen „szenographische Kompetenzen“"118 zum Tragen kommen, der logische Gegenpart zu Überwachungsinstrumenten wie Evaluationen und Projektbesuchen; potemkinsche Dörfer wurden auch Nyerere während der Umsiedlungskampagne immer wieder vorgesetzt. ${ }^{119}$ Eine GTZ-Expertin berichtete, dass sie vor einem NyerereBesuch in ihrem Bildungsprojekt von tansanischen Beamten instruiert worden sei, vor dem Präsidenten keinerlei kritische Bemerkungen fallen zu lassen. ${ }^{120}$

Auch der tansanische Counterpart-Koordinator, der als „Verbindungsoffizier“ zwischen tansanischen Behörden und TIRDEP fungierte, war ein solcher Kulturund Entwicklungsmakler, der vermittelte, Informationen filterte, Konflikte entschärfte und flexibel auf verschiedene Diskurse zurückgriff, um die Interessen der verschiedenen Seiten so auszutarieren, dass am Ende der Ressourcenfluss perpetuiert werden konnte. Wie sein deutscher Counterpart musste er ein Gespür für Machtverhältnisse und politische Konjunkturen haben. Auf tansanischer Seite lehnten einige Personen ab, als ihnen eine hohe Counterpartposition angeboten wurde, weil sie dem Druck von mehreren Seiten nicht ausgesetzt sein wollten. Der Posten verlangte einem ab, ein Universalist (jack of all trades) zu sein, um die Interessen der eigenen „Bosse“ in der Regierung, der „Deutschen“ und der Bevölkerung auszubalancieren, was mit einer Menge Druck einhergegangen sei, wie sich ein Beamter erinnerte, der diese Rolle eingenommen hatte: „You have to be a firm person“. ${ }^{121}$

116 Interview \#112, GTZ-Experte.

117 Privatarchiv S. R., [ohne Ordner], Deltgen, Interview mit Bundesminister Hans Klein, o.O., o.D. [1988], S. 4

118 Bierschenk u.a., Local Development Brokers, S. 22.

119 Schneider, Government of Development, S. 133-136.

120 Interview \#6, GTZ-Projektleiterin.

121 Interview \#77, Tansanischer Community Development-Experte; Interview \#80, Tansanischer Landwirtschaftsexperte; Interview \#84, Tansanischer Entwicklungsplaner und Counterpart. Wie einige der interviewten Counterparts im Interview meinten, profitierten sie in persönlicher Hinsicht kaum materiell von ihrer Position (z. B. stellte die GTZ bisweilen Transport, Unterkünfte und Koch, sodass keine Tagessätze anfielen), gewannen aber deutlich an Prestige. 
Dem externen Evaluierer Juma Ngasongwa zufolge war der höchste tansanische Counterpart in TIRDEP hauptsächlich ein ,public relations officer for the GTZ experts“. ${ }^{122}$ Es ging darum, Geberkonzepte in Tansania zu „verkaufen“, wie auch der Beamte, der diese Position eingenommen hatte, bestätigte:

I learned a lot through reading literature from DSE, from DAAD, from various organisations in Germany. And, okay, they are working in Cameroon for example. And this is their approach. Why don't you take that and try it here? My role is to convince my local colleagues and communities whether it can be adopted. ${ }^{123}$

Seine Funktion war aber keinesfalls auf diese einseitige Überzeugungsarbeit beschränkt. Er sah seine Rolle vor allem in Informationsübermittlung, der Überzeugungsarbeit in alle Richtungen; wenngleich aufgrund der höheren Wahrscheinlichkeit einer Implementierung freilich vor allem die Ansätze der Geberorganisation propagiert wurden. Hinzu kam eine Dienstleistungsfunktion für einen reibungslosen Alltag der expatriates:

You know, they are foreigners, so you have to support them in every area. Housing, visa, communication with the masses, communication with the leaders at the top. So you need to make the donor team rather comfortable. ${ }^{124}$

Um die Fortführung der Kooperation zu gewährleisten argumentierte er auch mit historischen Stufenmodellen und leitete aus ihnen eine entwicklungspolitische Sinnhaftigkeit fortgesetzter materieller Unterstützung ab:

I was always arguing with them. It took Europe about one hundred years from a cultural revolution to Industrial Revolution. Another one hundred years to reach the stage you are now. Now, in our situation you want us to change within five years, this is not possible. You introduce dairy development today, you want someone to be in a good position ... within five years, it's not possible. So you have to move in a gradual manner. Social change takes time. ${ }^{125}$

„Rückständigkeit“ war also Verhandlungsargument. Hinter diesem spezifischen Motiv steckte die allgemeinere Strategie, das Ende von Projekten möglichst lang

122 Ngasongwa, Evaluation, S. 300

123 Interview \#7, Tansanischer Entwicklungsplaner und Counterpart; Hervorhebung E. B.

124 Ebd. Für den Umgang mit expatriates und Statusunterschieden konnte er auch auf biographische Erfahrungen zurückgreifen: sein Vater war leitender Angestellter einer griechischen Firma gewesen, die Sisalplantagen betrieben hatte und wo die oberen Managementposten allesamt von Europäern besetzt gewesen waren.

125 Ebd. 
hinauszuzögern, wie er im Interview anhand mehrerer Beispiele ausführlich erläuterte. In einem Fall beruhigte er den deutschen Manager eines Projekts zur Kleinindustrieförderung, nachdem ein Vorhaben sich gegen dessen Willen entwickelt hatte: Eine Gruppe junger TansanierInnen hatte Materialien zur Schuhproduktion zur Verfügung gestellt bekommen, die Schuhe tatsächlich hergestellt, verkauft und war dann plötzlich mitsamt den erzielten Einnahmen von der Bildfläche verschwunden. Der deutsche Manager wütete über dieses vermeintlich kurzsichtige Handeln, in der Erinnerung seines tansanischen Counterparts drohte er:

\footnotetext{
"I am closing the project, there is no need if this is the trend. I want them to develop, I want them to accumulate money so that they can be a big company." I told him, "Wait, slowly. Take them step by step.” [...] He doesn't understand that in our society, if you get some money, you have to disappear for some time, you should spend the money (ukale), and then you come back. [...] It will take us more than three hundred years to develop to reach that level which you are today. ${ }^{126}$
}

Der tansanische Planer verwies also auf die andere Logik, um auch diesen Umgang mit Geld als rational darzustellen. Im Zuge seiner Vermittlertätigkeit sei die Gruppe nicht nur gefunden worden, sondern habe danach sogar an einem Seminar teilnehmen dürfen, in dem unternehmerische Fähigkeiten und längerfristige Profitorientierung vermittelt wurden. In einem zweiten Beispiel erläuterte der Planungsexperte sein Plädoyer für die Fortsetzung eines Milchwirtschaftsprojekts bis zur Etablierung einer vollständigen Vermarktungskette (hier konnten dann niederländische Ressourcen eingesetzt werden). Das dritte Beispiel stammt ebenfalls aus einem Milchwirtschaftsprojekt mit niederländischer Unterstützung, das Ende der 1980er-Jahre in Tanga begann und GTZ-Maßnahmen komplementierte. Hier intervenierte er als Counterpart in einen Konflikt zwischen „Experte“ und „Farmer“. Dem Bauern sei auf Kreditbasis vom Projekt eine junge Kuh zur Verfügung gestellt worden, er habe das Tier vernachlässigt, woraufhin es der niederländische Experte als sein Recht angesehen habe, die Kuh wieder zurückzuholen. (Die Dynamik ist übertragbar, die gleiche strafende und pädagogisierende Disziplinierungsmaßnahme findet sich, geschildert aus deutscher

126 Interview \#84, Tansanischer Entwicklungsplaner und Counterpart. Im Original: "Haelewi kwamba society ya kwetu huku ukishapata pesa you have to disappear for sometime ukale mpaka iishe ndio urudi. Akasema, ,Basi, I am closing the project there is no need if this is the trend, I want them to develop, I want them to accumulate money so that they can be a big company.' Nikamwambia, ,Wait, slowly. Take them step by step.”” 
Perspektive, auch in einem GTZ-Projekt. ${ }^{127}$ ). Wie im ersten Beispiel plädierte der Planer auch hier für eine Fortbildung, damit der tiergerechte Umgang sichergestellt werden konnte.

Diese Beispiele können und sollen hier nicht als Rekonstruktion der Aushandlungsprozesse aufscheinen, wie sie tatsächlich abgelaufen sind. Sie illustrieren jedoch erstens, wie Verweise auf kulturelle, historische, lebensweltliche Differenzen, die die „Deutschen“ vermeintlich „nicht verstanden“, dazu dienten, Ressourcenflüsse zu perpetuieren. Die Übersetzungsleistung bestand darin, den Deutschen die Handlungen und ihre zugrunde liegenden Motive so zu „kulturalisieren“, dass diese nicht zur Eskalation von Konflikten oder gar zum Abbruch der Ressourcenflüsse führten. Das bedurfte der Konstruktion der rückständigen Andersartigkeit ebenso wie dem Verweis auf die potenzielle Veränderbarkeit in Richtung des vom expatriate vertretenen Ideals - eine Kontinuität von Kolonialund Entwicklungsdiskursen. Zweitens sollten negative Konsequenzen der Disziplinierungsmaßnahmen für die „Zielgruppen“ verhindert oder rückgängig gemacht werden. Es war also kein reines, bürokratisches Eigeninteresse. Drittens wird deutlich, dass die Lösung „kultureller Probleme“, die bei der Übertragung technischer Modelle auftraten, schon vorgegeben war. Der Vermittlungsprozess ging von einem Konsens über die „Rückständigkeit“ der „Zielgruppen“ aus, es bestand Einvernehmen darüber, dass Rationalität und Verhalten in den „Zielgruppen“ mangelhaft waren. Das Unwissen wurde also auf Seite der „Zielgruppen“ verortet. Gleichzeitig „bildete“ der Planer mit seinen Interventionen aber auch die expatriates, indem er alternative Disziplinierungsmethoden zum strafenden, absoluten Entzug der Unterstützung aufzeigte. Was das gewünschte „Zielverhalten“ anging, wurde die Konzepthoheit der Geber aber nicht angetastet. Gleichzeitig verweisen die Beispiele auf einen Eigen-Sinn in den „Zielgruppen“, da sich viele Personen keineswegs einfach den Vorgaben unterwarfen, sondern sich die angebotenen Ressourcen in einer Art und Weise aneigneten, die mit den Projektzielen divergierten. Diese Spielräume wurden gerade auch durch die Tätigkeit der Entwicklungsmakler eröffnet.

127 Privatarchiv S. R., [PESP-Movies], Scheffler, Exposé für PESP-Filmreportage „Ein gemeinsamer Weg - Umweltangepasste Primarschulerziehung in Tansania“, o.O., 4.4.85, S. 14. Die betreffende Stelle im Exposé beruht anscheinend auf einer typischen Erfahrung und Umgangsstrategie. Zwei ExpertInnen werden mit den Worten zitiert: R.: „Die haben ziemlich dumm geguckt, sie hatten wohl nicht damit gerechnet, dass wir die Kuh wieder abholen.“ W.: „Macht nichts, wir können uns nicht alles gefallen lassen. Die Kuh kommt morgen nach Mwei.“ 


\section{Investoren: Die Inwertsetzung der Arbeitskräfte und Paternalismus wider Willen}

Der in der Frühphase des TIRDEP-Programms involvierte Volkswirt Rolf Hofmeier mahnte, „auf keinen Fall ständig alle Dinge selbst in die Hand nehmen, auch wenn immer wieder eine Versuchung dazu besteht“. ${ }^{128}$ Ideale der „Partnerschaft“ und „Zusammenarbeit“ mutierten in der Praxis jedoch häufig zu Alleingängen der expatriates, sodass der „deutsche“ Charakter des Programms bis zum Schluss klar erkenntlich blieb. ${ }^{129}$ Ein ehemaliger GTZ-Experte gestand im Interview, dass er der mühsamen Zusammenarbeit mit den Counterparts in den Beamtenstuben möglichst aus dem Weg gegangen sei. Er habe bevorzugt, direkt in die Dörfer zu gehen, den Treffen der Dorfgemeinschaft beizuwohnen und „zu[zu]hören, was die unter dem Mangobaum besprechen“. ${ }^{130}$ Das stand dem Partnerschaftsimperativ, der die Einbeziehung der Counterparts in den regionalen und Distriktbehörden verlangte, allerdings entgegen. Die expatriates von TIRDEP orientierten sich zur Bewertung ihrer Tätigkeit durchaus am Partnerschaftsideal - interpretierten dieses aber unterschiedlich. Der soeben zitierte ehemalige GTZ-Experte bezog sich im Interview auf das offizielle Skript, als er sagte, er „hätte die vielleicht aktiv aufsuchen müssen, die in Wert setzen - das ist uns wichtig, dass du mit uns zusammen arbeitest und so was“. ${ }^{131}$ Inwertsetzung (frz. mise-en-valeur) hatte in der Kolonialzeit bedeutet, die Kolonien durch Investitionen in die physische und soziale Infrastruktur ausbeutbar zu machen und so die Profite der Metropole zu erhöhen. Die postkoloniale Variante der Inwertsetzung ließe sich durchaus als neokoloniale Fortsetzung dieser Profitlogik deuten; dem zitierten Experten zufolge jedoch handelte es sich hierbei um ,eine Arbeitsbeziehung, die die Leute in die Lage versetzt hat, das zu tun, wofür sie auch da waren“, sprich, ihre Arbeitskraft im Allgemeinen und ihre fachlichen Qualifikationen im Besonderen $\mathrm{zu}$ entwicklungspolitischen Zwecken zu nutzen. ${ }^{132}$

Deutsche InterviewpartnerInnen beschrieben die „Inwertsetzung“ als mühsamen, schwierigen, oft gescheiterten Prozess, der ,in manchen Fällen [...] toll funktioniert“ habe, während man in anderen „betrogen“ worden sei. ${ }^{133}$ Die

128 Hofmeier, Die Tanga-Region, S. 327-328

129 Privatarchiv S. R., [BMZ-Evaluierung 1991 + TIRDEP Selbstevaluierung], S.R, Discussion Paper on Rural Development, Juli 1991, S. 25.

130 Interview \#26, GTZ-Experte.

131 Ebd.

132 Ebd.

133 Ebd. 
Inwertsetzung, im doppelten Sinn von symbolischer Wertschätzung und Nutzung der Arbeitskraft, war ein „wunder Punkt“ der Erinnerungen eines Projektleiters:

\begin{abstract}
Also bei [dem ersten Counterpart] habe ich nicht die Geduld aufgebracht, um ihn wirklich voll einschalten zu können. Es war auch objektiv nicht möglich, weil er einfach nicht konnte, nicht wollte. Und überhaupt kein Interesse hatte, sondern nur abstauben wollte, nicht. Das war so mein Eindruck. Aber man kann dann natürlich immer sagen, du hättest es anders anfassen sollen, vielleicht wäre es gelungen, ich glaube nicht. [...] [Der zweite Counterpart] war besser, der hat ja wenigstens mal eine Meinung von sich gegeben. ${ }^{134}$
\end{abstract}

GTZ-ExpertInnen stellten dem Eingeständnis der gescheiterten Kooperation auf Augenhöhe normalerweise eine Formel voran, die den eigenen Willen zur Partnerschaft und das „ständig[e] und aufrichtig[e] Bemühens des deutschen Teams um eine echte Partnerschaft in allen Projektbelangen“ bezeugte, das aber „in der Praxis immer wieder an der „Neigung“ des tansanischen Führungspersonals gescheitert sei, „Projektaktivitäten eben primär als ,deutsche“ und nicht automatisch als gemeinsame Maßnahmen anzusehen“. ${ }^{135}$ Das „Bemühen“ scheiterte in dieser Perspektive nicht an falschen Arrangements, Machtstrukturen oder eigenem Fehlverhalten, sondern an Unwillen und Unwissen der Counterparts. Diese selektive Kausalität fügte sich in die Fremd- und Selbstbilder, die bei EntwicklungsarbeiterInnen gängig waren und die eigene Präsenz nicht infrage stellten, sondern sie legitimierten. Mögliche strukturelle Faktoren oder Motive für die bewusste Abwehr einer „Zusammenarbeit“, etwa das Beharren auf eigenen Positionen, die Vermeidung zusätzlicher Arbeit oder die Aufrechterhaltung der eigenen Einflusssphäre, blieben unreflektiert oder zumindest undurchsichtig. Nur in einem Interview mit einem ehemaligen GTZ-Experten wurde ausnahmsweise ein rationaler Grund angegeben: Ein tansanischer Beamter, der von Ujamaa offensichtlich wenig hielt, warf seinem GTZ-Kollegen vor, zu viel zu arbeiten und damit den überfälligen Umsturz des Nyerere-Regimes hinauszuzögern. ${ }^{136}$

Die Spannung zwischen einer exekutiven und einer beratenden Rolle (siehe Kapitel 5.4) vor dem Hintergrund von Ressourcenaneignungsstrategien kennzeichnete auch die TIRDEP-Praxis. Ein Projektleiter klagte über die divergierenden Anforderungen von deutschen und tansanischen Stellen:

134 Interview \#25, GTZ-Projektleiter.

135 Hofmeier, Die Tanga-Region, S. 326. Siehe für weitere Beispiele Hofmeier, Die Tanga-Region, S. 317-318; Tanga Library, [General Correspondences], o.A. [VDP Project Manager?], Comments to Mr. Mahawi's Safari Report, o.O., 3.1.1983; vgl. auch ebd., [VDP Reports], VDP, Project Progress Report No. 8, Tanga, 20.6.1985, S. 3.

136 Interview \#102, GTZ-Experte. 
While the latter [Regional Development Director and Regional Agricultural Development Officer] are expecting a kind of commodity aid combined with management by the expatriates, the former [GTZ, BMZ] are providing 'advisory assistance' to 'Counterpart Organisation' which they suppose project implementors. The day-to-day project policy [...] consists in balancing the scarce resources of an advisory function with the expectations by the counterpart organisation of management and implementation by the project. ${ }^{137}$

Mit manchen Counterparts mochte die Zusammenarbeit über Jahre hinweg gut funktionieren, andere hingegen unterliefen die Erwartungen von GTZ-KollegInnen, die sich dann ,alleingelassen“ fühlten:

Wenn man über Zusammenarbeit redet, es wurde praktisch- Wurde man alleingelassen. [...] Der hatte ja keinerlei Fachkenntnisse. Hat auch gar nicht eingegriffen, hat nie ein Wort zu irgendetwas gesagt. Der wollte die Programme gar nicht sehen. Der wollte immer verreisen, von mir ein Auto haben, so was. Aber- Ist natürlich mitgefahren, als wir Europareisen- (lacht) Das alles gemacht, nicht. Aber der hatte keinerlei fachliche Funktion. ${ }^{138}$

Die Strategie des hier erwähnten Counterparts war offensichtlich, Reibungsflächen gar nicht erst entstehen zu lassen (auch die Versuche anderer ProjektleiterInnen, ihn zu konsultieren, prallten an seiner Meinung ab, dass ein Treffen gar nicht notwendig sei, „everything goes all right“ war seine höfliche Begründung ${ }^{139}$ ), wodurch sich der Projektleiter „alleingelassen“ fühlte. Ähnliche Erfahrungen, „gegen Watte gelaufen“ zu sein, machten auch andere ExpertInnen. ${ }^{140}$ Die GTZ-ExpertInnen handelten in solchen Fällen üblicherweise nach dem Muster, weitere Kooperationsversuche zu unternehmen und abzuwarten - allerdings zunehmend frustriert. Denn wenn der Counterpart nach wie vor als unwillig oder unfähig erschien, griffen Mittelabfluss- und Ergebnisdruck und die damit verbundene Notwendigkeit, an die eigene Karriere zu denken. Schließlich wurden die anstehenden Aufgaben einfach ohne Einbeziehung von Counterparts angegangen und abgewickelt.

Die Gründe für diese Dynamik sind in Rollenverständnissen und Diskursstrukturen sowie in den Macht- und Interessenlagen zu suchen. Der „Paternalismus der Partnerschaft“"141 war häufig nicht Voraussetzung, sondern Ergebnis einer gescheiterten Zusammenarbeit. Für viele GTZ-ExpertInnen in TIRDEP war es

137 BMZ, B 213/48195, RADO Heft 13, Project Manager Small Scale Irrigation Project to Regional Development Director, Fifth Half Yearly Progress Report, Tanga, 19.12.1986, S. 2.

138 Interview \#25, GTZ-Projektleiter.

139 Tanga Library, [General Correspondences], VDP Project Manager an TIRDEP Coordinator, Tanga, 18.5.1987.

140 Interview \#49, GTZ-Projektleiterin.

141 Eriksson Baaz, Paternalism of Partnership. 
ein Paternalismus wider Willen, eine Hierarchiebeziehung, die sie überwinden wollten, ohne zu wissen, wie. Die Statusunterschiede waren durch formelle Bestimmungen natürlich nicht nivelliert: Die unterschiedlichen Befugnisse und der ungleiche Zugang $\mathrm{zu}$ Ressourcen und Entscheidungsmacht beeinflussten zwangsläufig auch die „Kooperation“. Von einem tansanischen Beamten, der seinem GTZ-Counterpart theoretisch ebenbürtig gewesen wäre, hieß es hierzu:

Nobody was controlling. But of course the project manager, he is the one, he is the expert. So he is the one who is giving guidance. And then the others are giving out their ideas. ${ }^{142}$

Obwohl die „Kontrolle“ also auf die Schultern mehrerer Personen verteilt war, lag es doch beim (deutschen) Experten, die generelle Richtung vorzugeben. Versuche, jüngere Counterparts mit niedrigem Status zu fördern - hier kann man durchaus von Patronageverhältnissen sprechen, die manchmal auch in die private Sphäre hineinreichten - wurden von GTZ-ExpertInnen nicht als Pflicht geschildert, sondern als besonderes persönliches Engagement gedeutet, dass dann aber auch zu Enttäuschungen führen konnte:

Wir hatten ja zu [dem Counterpart] J. und seiner Familie ein relativ enges Verhältnis. ... Auch zu seiner Förderung - ich hatte ihn eigentlich so eingeschätzt, dass er noch ein bisschen mehr daraus machen kann, aus seinen Möglichkeiten. Und [ich habe ihn] deswegen auch nach England [geschickt]. Ich hätte die gerne dazu geführt, dass sie eigenständig werden. Und wir haben den ersten Schritt dazu auch gemacht. [...] Aber leider haben sie dann das Verhalten gezeigt, dass so wie [im Projekt] das Geld eben halt aus Deutschland kam [...]. ${ }^{143}$

Das Zitat belegt die Selbstverortung in einer Lehrerrolle sowie die damit verbundene emotionale Abhängigkeit. Der eigene Lehrerfolg definierte sich zwangsläufig nur über den Lernerfolg der SchülerInnen; der Misserfolg wurde hier ganz besonders deutlich, weil die problematischen Extraversionsstrategien und Abhängigkeitsbeziehungen sich nun auch in persönlichen Beziehungen offenbarten. Interviewte TansanierInnen hingegen sahen die „Inwertsetzung“ nicht in Aspekten der Counterpartbeziehungen, sondern vor allem als eine Frage von Disziplinregimes und Ressourceninputs, die im Folgenden genauer diskutiert werden.

142 Interview \#81, Tansanischer Bildungsexperte und Counterpart.

143 Interview \#115, GTZ-Experte. 


\subsection{Disziplinierungsketten}

TIRDEP-Projekte beruhten auf einer Reihe von Disziplinierungsmethoden. Manche Maßnahmen zielten auf eine kontinuierliche Überwachung der Arbeitsleistung, darunter Projektplanungsmatrizen mit Ergebnisübersichten, Arbeitspläne (ratiba) auf wöchentlicher und monatlicher Basis; wöchentliche Teamsitzungen, auf denen Ergebnisse präsentiert und (im Stile öffentlicher Selbstkritik) Erklärungen für Verzögerungen vorgebracht und die Aufgaben für die kommende Woche verteilt wurden. Wie alle tansanischen InterviewpartnerInnen übereinstimmten, war ein derart eng geknüpftes Disziplinregime in tansanischen Behörden, wo es Jahres- und bestenfalls Monats-, aber keine Tages- und Wochenpläne gab, höchst ungewöhnlich. ${ }^{144}$ Maßnahmen wie die strikte Kontrolle von Rechnungen oder die Überprüfung mittels Fahrtenbüchern und Stand der Kilometerzähler, ob das verbrauchte Benzin tatsächlich dem Arbeitsweg entsprach (privat gefahrene Kilometer mussten extra bezahlt werden,) reihten sich ein in weitere Mikromanagement-Techniken, mit denen die Handlungen und Bewegungen des tansanischen Personals auch auf Distanz kontrolliert werden konnten. Das führte auch zu entsprechenden Effekten der Selbstüberwachung:

Das bringt einen dazu, vorsichtig zu sein. Es gab auch die Leute, die für monitoring and evaluation zuständig waren. [...] Die überwachen einen. ${ }^{145}$

Die strenge Kontrolle der Ressourcen wurde auch als Vorteil gesehen, weil außerhalb von Projektkontexten die Wahrscheinlichkeit höher war, dass die öffentlichen Gelder in private Kanäle umgeleitet wurden. ${ }^{146}$ Ein tansanischer Techniker betonte, dass die Pläne und Vorgaben unabhängig von Status eingehalten wurden: Selbst eine „unwichtige Person wie ich“ (mtu mdogo kama mimi)

144 Interview \#51, Tansanischer Techniker und Abteilungsleiter: „Sisi tulikuwa monitored vizuri sana. Yani kila kitu kinakuwa recorded na kila jumatatu unatoa report ya kazi yako na unaandaa program for that week. Mnataka kufanya nini, na kama kwa wiki haitoshi mnahitaji wiki mbili na kila mwezi unasubmit ya mwezi. Kwa hiyo ni vitu ambavyo ambavyo katika hali ya kawaida, kwenye hizi organization za kawaida hizi za kiselikali yani ni mara chache sana.“

145 Interview \#58, Tansanische Gartenbauexpertin. Im Original: „Kwa hiyo hizo zinamfanya mtu uwe care[ful]. Kuna watu na monitoring and evaluation. ... Watu wanamonitor.“

146 Interview \#56, Tansanischer Landwirtschaftsexperte, Counterpart und späterer Projektmanager; Interview \#80, Tansanischer Landwirtschaftsexperte: „Most of the persons who were working with the project, they were different. Because in the project we had something called the project planning matrix. And the project planning matrix has activities, indicators, related to the targets. Lifespan of the project. You have to produce. And people had to report daily, weekly, monthly, half-yearly, yearly. Which is not the same as in the government offices. In the government offices, you have the annual plan. People do not have even the weekly plan.“ 
wurde vom Projektleiter erst um Erlaubnis gefragt, ob er den Wagen benutzen durfte, der eigentlich für den Techniker eingeplant geworden war. „Das Prozedere wurde respektiert“ (utaratibu uliheshimiwa), wie er meinte. ${ }^{147}$ Im Folgenden wird diskutiert, wie der modus vivendi in den disziplinarischen Arrangements der Projekte aus verschiedenen Perspektiven wahrgenommen wurde.

\section{Hybride Disziplinierungspraktiken}

Verknüpft man die Perspektiven der Beteiligten, erscheinen die Disziplinierungspraktiken in TIRDEP unweigerlich als hybrid. Die GTZ-ExpertInnen schildern sie als flexibel, entbürokratisiert und stark personenabhängig - kurz, von deutschen Standards weit entfernt und dezidiert tansanisch - während die TansanierInnen sie als stark formalisiert, rigide den (vom „Projekt“) gesetzten Regeln folgend und damit als typisch deutsch einstuften. Mehrere tansanische Counterparts erwähnten im Interview, dass die Projekte durch deutsche Steuergelder finanziert wurden, was die Kontrollfunktion deutscher Entsandter als gerechtfertigt erscheinen ließ. Damit spiegelten sie eine übliche Legitimationsrhetorik der bundesdeutschen Entwicklungsarbeit: Die Kontrolle wurde nicht mit Bezug auf das Personal, sondern in Bezug auf den Bonner Haushalt rationalisiert. Kulturalistische und bisweilen rassistische Interpretationen in der Kontinuität kolonialer Diskurse waren damit aber keineswegs passé. Die meisten tansanischen InterviewpartnerInnen bestätigten das in Tansania weitverbreitete Klischee der arbeitsamen Deutschen und viele erwähnten, dass sie von den deutschen KollegInnen viel über „Qualitätsarbeit“, Disziplin und Planung gelernt hatten und selbst - als sie später wieder in „rein tansanischen“ Arbeitsumfeldern tätig waren - für ihre ungewöhnlich straffe, ergebnisorientierte Arbeitshaltung geschätzt wurden. ${ }^{148}$ Mehrere deutsche TIRDEP-ExpertInnen hatten - wiederum an tansanische Schilderungen von Kolonialdeutschen erinnernd - den Ruf „strenge“ oder gar „aggressive“ (Sing. mkali, Plural wakali) Vorgesetzte oder KollegInnen zu sein, die die tansanischen Arbeitskräfte als faul und nicht arbeitswillig betrachteten; um die gesetzten Ziele zu erreichen, sorgten sie daher für „Druck“ (msukumo). ${ }^{149}$ Entschuldigungen und Ausreden für nicht vollbrachte Arbeitsleistungen wurden nicht akzeptiert, gleich ob es sich um kranke Angehörige handelte, man mehrere

147 Interview \#59, Tansanischer Landwirtschaftsexperte.

148 Etwa Interview \#51, Tansanischer Techniker und Abteilungsleiter; Interview \#80, Tansanischer Landwirtschaftsexperte. Der Ausdruck quality work stammt aus Interview \#7, Tansanischer Entwicklungsplaner.

149 Interview \#54, Tansanischer Fahrer. 
Stunden wartend in einer Schlange zugebracht hatte, um Zucker zu kaufen oder die privaten Einkommensstrategien Aufmerksamkeit erforderten - „die Deutschen“ wollten Ergebnisse sehen oder zumindest konkrete, praktische Lösungsvorschläge unterbreitet bekommen. ${ }^{150}$

I remember they were very serious with their work. Very serious. They follow up very closely, what we have agreed, they make a close follow-up. [...] Whenever you agree to deliver something, they help you. When you deliver, it's okay. But when you go the other way round, you can notice the colour of the face turns to red. ${ }^{151}$

Die Interviewnarrative tansanischer Fachkräfte vermitteln das Bild, dass die Etablierung des disziplinarischen Regimes auf Ebene der direkten Counterparts, des angestellten Personals und der sekundierten Fachkräfte, die materiell profitierten, weitgehend effektiv war. Mit zunehmender Entfernung von den Ressourcen- und Kontrollzentren nahm die disziplinierende Macht des Projekts jedoch ab. TIRDEP war kein Panoptikum, d.h. es konnten nicht alle assoziierten Arbeitskräfte überwacht werden, bzw. nicht allen das Gefühl vermittelt werden, überwacht zu werden, von der Einhaltung der Regeln zu profitieren oder bei deren Missachtung Sanktionen fürchten zu müssen. Viel eher war TIRDEP in Hinblick auf das disziplinarische Regime ein diffuses Feld, in dem Kräfte und Gegenkräfte wirkten und das Projektpersonal ebenso auf die „Zielgruppen“ reagierte wie umgekehrt.

Neben Repräsentationsketten zwischen lokaler Ebene und Entscheidungszentren, die durch Berichtpraktiken gebildet wurden, lassen sich auch Disziplinierungsketten erkennen. Eine besonders lange Kette etwa reichte vom GTZ-Projektleiter zu seinem Counterpart (einem tansanischen Beamten), von diesem zu einem Abteilungsleiter und von dort zu dem einfachen Beratungspersonal, das wiederum in direkter Interaktion mit Dorfautoritäten und -bewohnerInnen stand. Zwischen den einzelnen Scharnieren wurden die Anweisungen und Ziele nicht einfach eins zu eins übertragen, sondern je nach Eigeninteressen und Ressourcenverteilung gefiltert oder umgedeutet, unterlaufen oder verstärkt, ignoriert oder mit Eifer angeeignet.

In der Praxis der Disziplinierung griff deutsches Personal, gerade wenn es um das Aussprechen von Verwarnungen und Strafen ging, oft auf Counterparts zurück, die Arbeitskräfte mobilisieren sollten und zwischen den verschiedenen

150 Interview \#84, Tansanischer Entwicklungsplaner; Interview \#88, Tansanischer Landwirtschaftsexperte.

151 Interview \#16, Tansanischer Angestellter. 
Interessenlagen vermittelten. ${ }^{152}$ Sicht-, quantifizier- und messbare Ergebnisse waren die proklamierten Projektziele. Um die „richtigen“ Zahlen zu erreichen, übten die MitarbeiterInnen Druck auf Untergebene und die Bevölkerung aus. Einige TansanierInnen in solchen Positionen - loyal gegenüber deutschen wie tansanischen Vorgesetzten, strikt an Ergebnissen interessiert und furchtlos, unpopuläre Entscheidungen unnachgiebig „nach unten“ durchzusetzen - können als funktionale MittlerInnen gesehen werden, die den Plan auf die Implementierungsebene $\mathrm{zu}$ übersetzen hatten. Mehrere tansanische InterviewpartnerInnen meinten, dass „die Deutschen“ eine Vorliebe für jene Counterparts und Angestellten aufwiesen, die für das Projekt zählbare Ergebnisse brachten, aber von tansanischen KollegInnen als „harsch“ wahrgenommen wurden und von einer „aggressiven Ausdrucksweise“ (lugha kali) und Drohungen Gebrauch machten. ${ }^{153}$ Ein tansanischer Landwirtschaftsberater berichtete im Interview - offensichtlich stolz - über seine „strengen“, „harten“ (kali) Disziplinierungspraktiken. KollegInnen, die seiner Meinung nach nicht eifrig genug arbeiteten und die vorgegeben Ergebnisse nicht erreichten, habe er die Bezahlung vorenthalten, sie versetzen lassen und wenn notwendig Kontakte zu ParteifunktionärInnen, BeamtInnen und Polizeikräften aktiviert. ${ }^{154}$

Eine Studie der zwei Soziologen S. S. A. Ngware und B. C. Nindi von der Universität in Dar es Salaam zeigt, wie die Disziplinvorstellungen, die in den Büros in formuliert wurden, im Feld mit Eigen-Sinn ${ }^{155}$ unterlaufen wurden. Das Beratungspersonal (extension officers), das in die Dörfer ging, wurde teils von TIRDEP, teils vom Distrikt gestellt. Erstere absolvierten den Soziologen zufolge wöchentlichen Besuchen entsprechend ihren Plänen, zweitere besuchten weder die Dörfer noch hatten sie Arbeitspläne: „Some of them told us that they simply filled in TIRDEP's work implementation schedules without actually doing the work“. ${ }^{156}$ Angehörige dieser Gruppe beklagten sich, dass ihre Gehälter und Beförderungen nach wie vor vom Distrikt abhängig waren und sich TIRDEP nur für die Erfüllung eines Plans interessierte, sich aber nicht einmal ihrer Transport-

152 Bei den sekundierten Angestellten war das auch gar nicht anders möglich, da zwar die „technische“ Verantwortung beim Projekt liegen mochte, die „disziplinarische“ Verantwortung aber bei Regionalbehörde verblieb. Darüberhinaus intervenierten tansanische Counterparts aber auch in Fragen, die das Personal betrafen, das von TIRDEP direkt angestellt war.

153 Interview \#81, Tansanischer Beamter und Bildungsexperte.

154 Ähnlich auch Interview \#58, Tansanische Gartenbauexpertin.

155 Lindenberger, Herrschaft und Eigen-Sinn.

156 S. S. A. Ngware/B. C. Nindi, Sociological Study: „Soil Erosion Control, Agroforestry Project, and Livestock Development in the Western Usambaras“.. Prepared for TIRDEP, Dar es Salaam 1982, S. 51. 
probleme annahm. ${ }^{157}$ Hier bestand also kein Konsens. Diejenigen, die sich den Disziplinierungspraktiken unterwerfen sollten, sahen in dem breiteren Arrangement nicht genügend Vorteile und entzogen sich der Überwachung. ${ }^{158}$ Einige der sekundierten extension officers, so die beiden Soziologen weiter,

\begin{abstract}
shocked the survey team by openly complaining that the TIRDEP expatriate staff were very strict with the agreed work schedules and closely followed what the village extension workers were doing daily and weekly. This was done through filling in a form which was handed over to the TIRDEP staff during their weekly vis[i]ts to the villages. Such extension workers boasted they used to do very little when they were working directly under DDD [District Development Director] and had more time for leisure but with the same pay. One of them said he would be happier to be ,returned" to the DDD. They suggested that if TIRDEP projects were to be implemented properly, then they should employ ,their own people' as they did with their administrative staff at Lushoto or agree to ,topping up their salaries with fringe benefits. [...] Worse still, most of them had a very low morale, and kept on complaining that since ,TIRDEP had money, they should give us something more as an incentive to doing ,their ${ }^{6}$ work. [...] [T] hese workers (very unfortunately) thought that TIRDEP was too much of a bother to them because they demanded a particular work-schedule be implemented whereas (according to them) they could get the same pay by doing the minimum if they had been under the DDD, who, because of a number of constraints could not supervise every worker as closely as TIRDEP people did. ${ }^{159}$
\end{abstract}

In den (im Ton der Empörung verfassten) Ausführungen der beiden Soziologen zeigt sich deutlich, wie diejenigen Arbeitskräfte, die Zugang zu Projektressourcen wie Gehaltsaufstockungen und Lohnnebenleistungen hatten, eine größere Bereitschaft zeigten, ihre Arbeit dem Disziplinregime anzupassen, während jene extension officers, die nur sekundiert wurden und daher keinen Zugang zu Projektressourcen erhielten, die Ungleichbehandlung als ungerecht empfanden. Das Disziplin- und Umverteilungsregime des Projekts erscheint hier als Insel, die nur wenige Berührungspunkte mit dem Umfeld aufwies und sogar versuchte, diese zu minimieren. Aus der Perspektive der enttäuschten extension officers brachte TIRDEP für den eigenen Arbeitsalltag nur Nachteile, darunter eine stärkere Überwachung und höhere Arbeitsleistung; hatte aber nichts zu bieten, was den gesteigerten Aufwand und eingeschränkten Freiraum rechtfertigte. In einem Bildungsprojekt klagte die Projektleiterin, dass „nötige Disziplinarmaßnahmen nicht durchgeführt [wurden], wenn das Projekt nicht Druck ausübt[e]“. ${ }^{160}$ Was die

157 Ngware/Nindi, Sociological Study, S. 44-48.

158 Vgl. Dyck, Anthropological Perspectives on Discipline, S. 10.

159 Ngware/Nindi, Sociological Study, S. 49, 51, 63; Formatierung im Original.

160 Privatarchiv S. R., [Korrespondenz mit GTZ-HQ FB 221], S. R., M\&E-Erfahrungen im Projekt PESP, o.O., 13.1.1988. 
tansanischen SchulinspektorInnen anging, die für die Überwachung der einzelnen Schulen zuständig waren, so fand die Projektleiterin, müsse man die „praktisch nicht existente Supervision ihrer Arbeit im Rahmen ihrer eigenen Behörde [...] halt als Rahmenbedingung akzeptieren und durch die Projektarbeit zumindest für die Projektlaufzeit verbessern“. ${ }^{161}$ Als „Gegenleistung für die $\mathrm{Zu}$ satzarbeit“, die von der Projektleitung gefordert wurde, erhielten die Inspektoren Motorräder und Ersatzteilnachschub. ${ }^{162}$ Hier wurde also auf materielle Anreize zurückgegriffen.

Zumindest in Einzelfällen wirkte die Disziplinierungskette mit ihren verschiedenen Scharnieren, Filterungen und Kraftübersetzungen von „oben“ nach „unten“ verstärkend („A minor complaint from TIRDEP expatriate staff led [to] the extension workers getting hell from the District Authorities“163). In die entgegengesetzte Richtung wurden die Klagen des Beratungspersonals, etwa mittels der soziologischen Studie, auf dem Weg nach „oben“ nicht nur abgeschwächt, sondern auch diskreditiert. Die beiden Soziologen vom Institute of Development Studies gingen nicht auf das Problem der ungleichen Entlohnung für gleiche Arbeitsleistung ein, sondern empfahlen politische Schulungen durch die Partei, die von TIRDEP finanziert werden sollten. Sie setzten also nicht auf materielle Anreize, sondern vertraten den politisch opportuneren Ansatz idealistischer Mobilisierung, der praktisch allerdings oft ineffektiv war. ${ }^{164}$ Hier spielten auch koloniale Hypotheken eine Rolle.

\section{Koloniale Hypotheken}

In der Beziehung des Projektpersonals zu den „Zielgruppen“ offenbarten sich die „Waffen der Schwachen“: alltägliche, auf sofortige Effekte ausgerichtete Widerstandsformen, mit denen die Disziplinierungsansprüche unterlaufen wurden. ${ }^{165}$ Im Umgang mit dem Staat - der z. B. die Abgabe landwirtschaftlicher Produkte zu niedrigen Preisen forderte - hatte die ländliche Bevölkerung eine Reihe von

161 Privatarchiv S. R., [Korrespondenz mit GTZ-HQ FB 221], S. an I., o.O., 8.1.1987.

162 Privatarchiv S. R., [Korrespondenz mit GTZ-HQ FB 221], S. R., M\&E-Erfahrungen im Projekt PESP, o.0., 13.1.1988.

163 Ngware/Nindi, Sociological Study, S. 48; siehe auch einen typischen Brief eines Parteivorgesetzten an einen Funtionär unterer Ebene, in dem eine Beschwerde von Gebern in hierarchischen Druck übersetzt wird: Tanga Library, [General Correspondence], Mkurugenzi Mtendaji (Halmashauri ya Wilaya Muheza) an Diwani ya Kata, Muheza, 22.6.1987.

164 Ngware/Nindi, Sociological Study, S. 83.

165 Scott, Weapons of the Weak. 
Umgehungsstrategien erprobt, um die eigene Subsistenz zu gewährleisten. Erst im Zuge des Paradigmenwechsels hin zu partizipativen Methoden aber erkannten GTZ-ExpertInnen nun ein dauerhaftes Problem in den „patriachalischen Verhaltensweisen gegenüber Zielgruppen“; gerade das Beratungspersonal, „das wichtigste Glied in der Kommunikationskette“, falle immer wieder in ein „Predigen“ zurück, statt auf die Bedürfnisse der „Zielgruppen“ einzugehen. ${ }^{166}$ Das Verhalten der tansanischen Fachkräfte der untersten Ebene - also gerade jener Personen, die am häufigsten in den direkten Kontakt mit den „Zielgruppen“ traten - wurde als autoritär, rechthaberisch und besserwisserisch beschrieben. ${ }^{167}$ Die mit den Beratungsdienstleistungen „zufriedensten“ Personen trafen die Soziologen Ngware und Nindi ausgerechnet in jenen Dörfern an, wo das Beratungspersonal keinen Druck ausübte („did not bother them very much“) oder gar nicht erst aufkreuzte. ${ }^{168}$ Anderenfalls standen den „Zielgruppen“ bewährte Strategien zur Verfügung, ihrer landwirtschaftlichen Produktion wie zuvor nachzugehen. Wo Dorfautoritäten und externes Beratungspersonal divergierende Anweisungen gaben, entschieden sich die BewohnerInnen für die sicherste Strategie, „to ignore both and remain safe.“ Neben Formen passiven Widerstands - „listen, nod and everything ends there“ - gab es auch offene Kritik mit Bezug auf eigene Erfahrungen in der Vergangenheit; gerade ältere DorfbewohnerInnen kritisierten die Methoden des Beratungspersonals als Wiederkehr kolonialer Interventionen und Zwänge. ${ }^{169}$

Die koloniale Hypothek wog gerade in den Usambara-Bergen schwer. Die dort angesiedelten TIRDEP-Projekte ${ }^{170}$ mussten sich von den Zwangsmethoden der Kolonialzeit abgrenzen, wenn sie Aussicht auf Erfolg haben wollten. Auf dringende Empfehlung lokaler Behörden wurden von GTZ-Entsandten angedachte Maßnahmen wie das arbeitsintensive Anlegen von Terrassierungen, die zur

166 Tanga Library, [Progress Reports], GTZ, Stellungnahme zu VDP Fortschrittsbericht Nr. 5, Eschborn, o.D. [1991], S. 2; BMZ, B 213/48197, RADO Heft 14, GTZ FB 132, Stellungnahme zu Fortschrittsberichten, 30.8.1988.

167 Weitere Studien über die Landwirtschaftsberater, deren Ergebnisse mit den Schilderungen von Ngware und Nindi weitgehend übereinstimmen, sind: Tanga Library, TIR 9a/4, Monitoring and Evaluation Unit, Bwana Shambas‘ Annual Report (1981), Tanga, Dezember 1982; ebd., TIR PAM 0053, TIRDEP Monitoring and Evaluation Unit, Agriculture Extension Performance Survey, Tanga, Oktober 1982; B. C. Nindi, Traditional Agricultural Extension System in Tanzania: A Critical Analysis, in: Journal of Eastern Africa Research and Development 16 (1986), S. 122-132.

168 Ngware/Nindi, Sociological Study, S. 52, 54, 60-63.

169 Ebd., S. 200.

170 Das Livestock Development Project (LDP) begann 1979 und wurde mit dem 1981 gegründeten Soil Erosion and Control and Agroforestry Project (SECAP) unter dem Namen des letzten zusammenlegt. Dieses erweiterte SECAP lief von 1984 bis 2000. 
britischen Kolonialzeit zu Aufständen geführt hatten, (zumindest anfangs) wieder verworfen. ${ }^{171}$ Die Beziehungen zwischen der ländlichen Bevölkerung dieser Region und den direkt sichtbaren Repräsentanten des Staates, dem lokalem Beratungspersonal (agricultural extension officers, bwana shamba), waren ebenfalls historisch vorbelastet. ${ }^{172}$ Als im Distrikt Lushoto u.a. auf Drängen der GTZ-Experten auch wieder Verordnungen ähnlich wie in der Kolonialzeit erlassen wurden, mühte sich die GTZ festzustellen, dass diese - allen Ähnlichkeiten zum Trotz - „nicht mit den Zwangsmaßnahmen zu verwechseln [waren], die die britische Kolonialregierung in bester Absicht aber entgegen den Einsichten und Interessen der Bevölkerung mit voller Staatsgewalt durchsetzen wollte“. ${ }^{173}$ Als wichtigster Unterschied wurde angegeben, dass die neuen Regelungen „Spielraum“ ließen. Auffällig ist aber, dass in der Peripherie der Projektintervention zunehmend Sanktionsmechanismen zum Einsatz kamen; dass also Personal unterer Ebenen (im Gegensatz zu den höherrangigen Counterparts) fürchten musste, die materiellen Belohnungen eventuell nicht zu erhalten. Die „Zielgruppen“ wurden sogar direkten Strafen unterworfen: Für den Verstoß gegen Verordnungen, was z.B. das Unkrautjäten auf den eigenen Feldern anging, konnten ebenso wie für die Nicht-Teilnahme an kollektiven „Selbsthilfe-Aktionen“ Geldstrafen verhängt werden; in einigen Fällen gab es Festnahmen. ${ }^{174}$

Laut den Autoren der soziologischen Studie genossen GTZ-expatriates im Vergleich mit Kolonialbeamten einen guten Ruf: „[F]armers pointed out a big contrast between the colonial expatriate staff whom they hated and feared and the present expatriate staff who were friendly and ready not only to help solve the farmers' development problems but also did not consider farmers as mere passive participants but considered them as active participants“. ${ }^{175}$ Das entspricht dem Selbstbild der meisten TIRDEP-ExpertInnen; es ließe sich allerdings vermuten,

171 Interview \#56, Tansanischer Landwirtschaftsexperte, Counterpart und späterer Projektmanager. Später wurden die Terrassierungen dann von manchen im Beratungspersonal befürwortet, aber das deutsche Projektmanagement sträubte sich mit Verweis auf den Widerstand dagegen in der Vergangenheit. Siehe Johannsson, Ten Million Trees Later, S. 87.

$172 \mathrm{Zu}$ den äußeren Interventionen während der Kolonialzeit und nach der Unabhängigkeit sowie der Verabschiedung von Verordnungen, die alle als schädlich betrachteten landwirtschaftlichen Praxen unter Strafe stellten, siehe im Detail Johansson, Ten Million Trees Later, S. 37-51; Michaela von Freyhold, Ujamaa Villages in Tanzania. Analysis of a Social Experiment, London 1979, S. 8-9, 35-43; Feierman, Peasant intellectuals, Kap. 2.

173 BMZ, B 213/48195, GTZ, Fachliche Stellungnahme zu Unterstützung des RADO, LRE-Programm Tanga, TIRDEP, Eschborn, den 25.8.1987, S. 5.

174 Interview \#16, Tansanischer Angestellter; Interview \#88, Tansanischer Landwirtschaftsexperte; Nindi, Traditional Agricultural Extension System, S. 122.

175 Ngware/Nindi, Sociological Study, S. 103. 
dass die Autoren hier den Auftraggebern der Studie auch schmeicheln wollten eine Kritik am Verhalten von GTZ-Personal oder der Interventionslogik findet sich auf keiner der über zweihundert Seiten der Studie.

Einen eigensinnigen Umgang mit den Projektzielen in den Dörfern konnten sich die wirtschaftlich Bessergestellten leisten, die - nicht unähnlich den kapitalintensiven Landwirtschaftsprojekten Anfang der 1960er-Jahre in Tanganjika als „progressive“ Modernisierungsvorbilder für die ärmere kleinbäuerliche Bevölkerung dienen sollten. ${ }^{176}$ Sie pflanzten das vom Projekt beworbene Guatemalagras nicht aus Überzeugung von der Maßnahme, die eigentlich auf Erosionsschutz abzielte, sondern nur, weil die Pflanzungen die Voraussetzung darstellten, um ein Rind auf Kreditbasis zu erhalten. ${ }^{177}$ Dieser Ansatz wurde später aufgegeben, aber er weist auf das generelle Problem der „Elite“-Orientierung hin. In institutioneller Hinsicht operierte das Projektpersonal vor allem mit den Bessergestellten; nicht zuletzt dadurch waren - in Hinblick auf die Ergebnisse - die Vorteile, die aus den Projekten gezogen werden konnten, ungleich verteilt, worüber die ausgegrenzten Mehrheiten wiederholt ihren Unmut bekundeten, während sich das Projektpersonal damit z.T. arrangierte und die wachsende Ungleichheit in Kauf nahm. ${ }^{178}$

\section{Die Akzeptanz der wachsenden Ungleichheit}

Der Matthäus-Effekt - „Wer hat, dem wird gegeben“ - war auch in Tanga zu konstatieren. Ein BMZ-Evaluierungsteam stellte 1982 mit Besorgnis fest, dass TIRDEP nur der „élite of the poor“ Akkumulationsmöglichkeiten bot und somit die Ungleichheit in lokalen Kontexten verschärfte. ${ }^{179}$ Die Evaluation war ein abgeschwächtes Echo der Ziele der 1970er Jahre, als „die Förderung der UjamaaBewegung und Beteiligung der Bevölkerung am Entwicklungsprogramm“ noch ganz oben auf der Liste der Ziele gestanden hatte - und zwar in einem Atemzug mit der Verbesserung der Lebensbedingungen sowie gleichmäßigerer Einkommensverteilung. ${ }^{180}$ Die Zielgruppendefinition von TIRDEP-Projekten hatte die „ländliche Bevölkerung“ allerdings lange als monolithische, undifferenzierte Gruppe behandelt. In der damals verbreiteten, moralisch geladenen

176 Johannsson, Ten Million Trees Later, S. 71.

177 Ebd., S. 86.

178 Ebd., S. 71.

179 Tanga Library, BMZ, Main Report on the inspection of the Technical Cooperation Project TIRDEP, Bonn, 1.12.1982, S. 69; Ngasongwa, Evaluation, S. 318, 497.

180 PAAA, ZW 114 925, BMZ, Vorlage zur Entscheidung, Bonn, 19.12.1973, S. 10. 
marxistischen Terminologie gesprochen, erreichte TIRDEP aber in erster Linie die in jedem Dorf zu findende Schicht der „Kulaken“, die außerfamiliäre Arbeitskräfte saisonal zur Bestellung ihrer Felder und Plantagen anstellten, kleine Geschäfte besaßen, Handel trieben und dank ihrer wirtschaftlich vergleichsweise privilegierten Position riskieren konnten, mit neuen und kapitalintensiven Produktionstechniken zu experimentieren. Hinzu kam, dass sie ihr ökonomisches Gewicht in politisches Kapital konvertieren konnten. Die Monopolisierung der politischen Macht ermöglichte dann auch die Aneignung der Vorteile, die durch eventuelle Entwicklungsprojekte ins Dorf kamen. ${ }^{181}$ Nur die Bessergestellten konnten das Risiko eingehen, in die abhängigkeitsstiftenden Kreditinitiativen der Projekte einzusteigen. All das stand den proklamierten Idealen des sozialistischen Entwicklungswegs entgegen: Nicht kommunale Produktion und das Wohl der ländlichen Bevölkerung im Sinne von Ujamaa wurden gestärkt, sondern privates Unternehmertum und Profitstreben von wenigen. ${ }^{182}$

Dem GTZ-Personal waren diese Aspekte bewusst; zumindest wurden sie gelegentlich bei Teammeetings und durch unregelmäßige externe Impulse ins Bewusstsein gerufen. Angestoßen etwa durch die breit rezipierte Kritik der ehemaligen BMZ-Beamten Brigitte Erler ${ }^{183}$, die vor allem die Verschärfung der sozialen Ungleichheit durch BRD-geförderte Entwicklungsprojekte kritisierte, überprüfte ein GTZ-Experte, dem die Erler-Kritik „doch etwas zu denken gegeben“ hatte, die einzelnen TIRDEP-Projekte ad hoc auf ihre „Verteilungswirkung“. Er kam zu dem Schluss, dass die drei Projekte in den Bereichen Gesundheit, Bildung und Wasser generell grundbedürfnisorientiert waren und sich die Frage der Verteilungsgerechtigkeit also gar nicht stelle (was ebenfalls zu hinterfragen ist, da die Standortwahl oft von privilegierten Gruppen beeinflusst wurde); für die produktionsorientierten Kleinindustrie- und Landwirtsprojekte konstatierte er eine eindeutig negative Wirkung („Hier verschlechtern wir die Einkommensverteilung“), setzte aber umgehend zur Rationalisierung an:

181 Siehe Freyhold, Ujamaa Villages, 19. In TIRDEP: BMZ, B 213/48197, Project Leader TIRDEP DRAP, Project Progress Report No. 14, Tanga, 30.6.1988.

182 So auch Ngasongwa, Evaluation, S. 497.

183 Belege für die Rezeption von Erlers Buch finden sich in: Privatarchiv S. R., [BMZ-EVALUIERUNG 1991 + TIRDEP SELBSTEVALUIERUNG], Memo von S. an Leiter der Koordinationsgruppe, Tanga, 6.6.1985; ebd., [KORRESPONDENZ MIT GTZ-HQ FB 221], Projektleiterin PESP, Beantwortung GTZ-Fragebogen zur Projektarbeit, o.O., o.D. (1986], S. 14. Das Buch war sehr begehrt und in der TIRDEP-Bibliothek ständig verliehen - s. ebd., [BIBLIOGR. \& ABSTRACTS]. 
Das schadet nichts, nicht alles, was TIRDEP macht, kann armutsorientiert sein. [...] Wer ,entwickelt', nimmt immer ku[r]z- bis mittelfristig Ungleichheiten in Kauf, sofern er in irgendeiner Form an das ökonomische Potential der Zielgruppen appeliert [sic]. ${ }^{184}$

Eine „kurzfristige“ Zunahme der Ungleichheit wurde mit dem Verweis auf eine „langfristige“ Entwicklung und Nachhaltigkeit legitimiert. Begonnen hatte TIRDEP mit dem Ziel, die Lebenslage - und wirtschaftliche Situation - der gesamten Bevölkerung, vor allem aber der ärmsten, ländlichen Bevölkerungsanteile zu verbessern. Die Akteure der bundesdeutschen Entwicklungsarbeit waren sich jedoch uneins, ob Tansanias Politik, in die die eigenen Maßnahmen eingebettet sein sollten, diesem Ziel dienen konnte. In Bonn und Eschborn wurde schon 1977 stark bezweifelt, dass die in Tansania offiziell diskreditierte „,kapitalistische“ Motivation, d.h. das Gewinnstreben des Einzelnen“ tatsächlich durch eine „andersgeartete Motivation“ ersetzt werden könnte. ${ }^{185}$ In einer BMZ-Evaluierung aus dem Jahr 1983 wurde es nun als „Fakt“ hingenommen, dass manche Projekte die ärmsten Bevölkerungsschichten überhaupt nicht erreichten. ${ }^{186}$ Eine ähnliche Dynamik offenbart sich in der regionalen Verteilung der Ressourcen: Zwar wurden ökonomisch schwächere Distrikte wie Handeni und Pangani anfangs bevorzugt, letztendlich flossen die meisten Mittel aber immer weniger in die Peripherie, sondern in erster Linie in die vergleichsweise bessergestellten Distrikte Tanga, Muheza und Lushoto. ${ }^{187}$ Abhilfe sollten nunmehr neue Planungs- und Umsetzungsmethoden schaffen, allen voran die Partizipation.

\section{Partizipation als Umgehung und Widerstand}

Die Zielorientierte Projektplanung (ZOPP) wurde in der zweiten Hälfte der 1980er Jahre von der GTZ sukzessive auf alle Projekte aufoktroyiert. Dieses neue, auf engen Mittel-Zweck-Relationen und Kausalitätsschemata beruhende Konzept der BRD-Entwicklungsarbeit bediente sich konzeptuell beim logical framework, das vom Pentagon entwickelt worden und erst von US-Wirtschaftseliten, dann in entwicklungspolitischen Kreisen aufgegriffen wurde. ${ }^{188}$ ZOPP sollte die

184 Privatarchiv S. R., [BMZ-Evaluierung 1991 + TIRDEP Selbstevaluierung], S. an E. (TIRDEP Projektkoordinator), Tanga, 6.6.1985.

185 BArch Koblenz, B 213/33065, Rosenthal (BMZ), Vermerk, Bonn, 29.3.1977, S. 4; siehe auch die GTZ-Arbeitspapiere in derselben Akte.

186 „The fact has to be accepted that the project [SECAP] has no specific programme for the poorest farmers.“ BMZ-Evaluation 1983, zit. nach Johannsson, Ten Million Trees Later, S. 71.

187 Ngasongwa, Evaluation, S. 407.

188 Köhler, Mittler zwischen den Welten, S. 117-120. 
Einbeziehung aller Beteiligten in Planung (und später auch Evaluierung) sicherstellen, insbesondere auch VertreterInnen der „Zielgruppen“. Die in den 1990er Jahren zum Mainstream gewordenen partizipativen Techniken im Entwicklungssektor sind in der Literatur einerseits als entpolitisierendes Kontrollinstrument mit nur rhetorisch statt substanziell vorhandener Mitbestimmung kritisiert worden; Gegenstimmen haben Partizipation als ambivalenten, aber grundsätzlich offenen Aushandlungsprozess dargestellt, der auch die Bearbeitung von Ungleichheiten erlaube. ${ }^{189}$ Beiden Argumenten fehlt jedoch die historische Dimension. Im Zusammenhang mit der Krise des Entwicklungsstaates ist daher die These des Politikwissenschaftlers Thandika Mkandawires nützlicher. Mkandawire hat „Partizipation“ als Strategie gefasst, mit der „externe Eliten“ (die expatriates) „lokale Eliten“ umgehen können:

A major preoccupation of external elites was the problem of ,elite capture' of state policies. [...] What we had here was an anti-elite elitism in which expatriate elites sought to circumvent their venal counterparts to directly deal with the ,grassroots' through participatory processes they funded and dominated. ${ }^{190}$

ZOPP, die bundesdeutsche Form des partizipativen Instrumentariums, sollte gerade dazu dienen, sowohl die Kluft zwischen expatriates und Counterparts einerseits als auch zwischen dem „Expertenwissen“ des (deutschen wie tansanischen) Projektpersonals und dem „lokalen Wissen“ der „Zielgruppen“ andererseits zu überwinden. TansanierInnen absolvierten die ZOPP-Fortbildungen und übernahmen sukzessive die einflussreiche und für Ergebnisse entscheidende Rolle des Moderierens (wenngleich deutsche GTZ-Kräfte die oft suggerierten Antworten und die schematische Anwendung der Methodik bekrittelten). Durch die neuen Konsultationstechniken konnten zuvor marginalisierte Gruppen

189 Zur Position, Partizipation sei mit offener Aushandlung verbunden, siehe Gabriele Beckmann, Partizipation in der Entwicklungszusammenarbeit. Mode, Methode oder politische Vision? Hamburg 1997; Trevor Parfitt, The Ambiguity of Participation. A Qualified Defence of Participatory Development, in: Third World Quarterly 25/3 (2004), S. 537-555; Glyn Williams, Evaluating Participatory Development. Tyranny, Power and (Re)Politicisation, in: Third World Quarterly 25/3 (2004), S. 557-578; Giles Mohan/Samuel Hickey, Hg., Participation, from Tyranny to Transformation? Exploring New Approaches to Participation in Development, London 2004. Zur kritischen Position, die den partizipativen Methoden Entpolitisierung und die Fortschreibung von Machtungleichheiten ankreidet, siehe Bill Cooke/Uma Kothari, Hg., Participation: the New Tyranny?, London, New York 2001; Ilan Kapoor, The Devil's in the Theory. A Critical Assessment of Robert Chambers' Work on Participatory Development, in: Third World Quarterly 23/1 (2002), S. $101-117$.

190 Thandika Mkandawire, Fifty Years of African Independence. Personal Reflections. Distinguished Nyerere Lecturer, Dar es Salaam 2013, S. 43. 
- insbesondere Frauen - ihre Standpunkte in mehreren Fällen darlegen, sodass ihre Prioritäten Berücksichtigung fanden und z. B. zum Bau eines Brunnens statt eines neuen Genossenschaftsgebäudes führten. Denkweisen und Argumente, die anders gar nicht zur Sprache gekommen wären, konnten so bisweilen sichtbar gemacht werden. Die Praxis hält aber auch Gegenbeispiele bereit: „Da saßen immer nur die Beamten“, erinnerte sich eine Projektleiterin, und in manchen ZOPP-Sessions wurden, „weil alles schnell gehen musste“, gar überhaupt keine TansanierInnen einbezogen. ${ }^{191}$

Der Versuch, mit Partizipationstechniken in den 1980er-Jahren einen direkten Zugang zu den „Zielgruppen“ zu erlangen, war Teil in der Selbstpositionierung eines wachsenden Anteils von expatriates als eine spezifische anti-elitäre Elite (allerdings unter anderen Vorzeichen als die politische Elite Tansania, die sich ebenfalls so positionierte). Die Frustration mit der tansanischen Bürokratie und Versuche, die offiziellen Stellen zu umgehen, führten in einigen TIRDEP-Projekten Ende der 1980er-Jahre zu einer ,vollständige[n] Hinwendung an die Zielgruppen (Dörfer, Gruppen, Individuen) unter (bewusster) Umgehung der staatlichen Träger“. ${ }^{192}$ Anfangs hatte TIRDEP den Ansatz verfolgt, durch die offiziellen tansanischen Institutionen $\mathrm{zu}$ arbeiten und diese durch die Kooperation zu stärken. ${ }^{193}$ Zunehmend zielten GTZ-ExpertInnen aber darauf ab, die Arbeit mit den Behörden und ,konservativen Dorfregierungen [...] auf das Notwendige [zu] beschränken [...] damit uns nicht das gleiche Misstrauen trifft“. ${ }^{194}$ Politische Eliten und Verwaltungskräfte galten nun meist als unbeliebt und nicht-repräsentativ. Sozialwissenschaftliche und kulturanthropologische Studien aus den 1980er Jahren legten nahe, dass die breite Bevölkerung von Entscheidungsprozessen und der Allokation von Resourcen vielfach ausgeschlossen blieb - selbst auf Dorfebene. ${ }^{195}$

191 Interview \#8, GTZ-Projektleiterin; siehe auch Privatarchiv S. R., [Team-Meeting Minutes \& Papers 1985-89, Bd. 1], Minutes of TIRDEP Team Meeting, o.O., 15.2.1988. Differenziertere Perspektiven, die dieser Erkenntnis aber nicht grundsätzlich entgegenstehen, finden sich in Dirk Kohnert u.a., Hg., Perspektiven zielorientierter Projektplanung in der Entwicklungszusammenarbeit, München 1992.

192 Privatarchiv S. R., [BMZ-Evaluierung 1991 + TIRDEP Selbstevaluierung], M. S. an Leiter der Koordinationsgruppe, Tanga, 7.3.1991.

193 BArch Koblenz, B 213/33065, Rosenthal (BMZ), Vermerk, Bonn, 29.3.1977; ebd., Rosenthal (BMZ), Vermerk zum Besuch des Regional Commissioner der Tanga-Region, Bonn, 1.4.1977; Tanga Library, [VDP Project Reports], VDP, Project Progress Report No. 11, Tanga, 20.1.1987.

194 Privatarchiv S. R., [BMZ-Evaluierung 1991 + TIRDEP Selbstevaluierung], Projektleiter VWSP an alle TIRDEP-Projektmanager, o.O., 4.4.1991; siehe auch Mhina, The Impact of TIRDEP, S. 47-48. 195 Ngasongwa, Evaluation, S. 386; Graham Thompson, The Bewitchment and Fall of a Village Politician, in: R. G. Abrahams, Hg., Villagers, Villages and the State in Modern Tanzania, Cambridge 1985, S. 110-134. 
Die Soziologen Ngware und Nindi wiesen in ihrer Studie auf bewusste Täuschung und Irreführung durch Parteikader und Dorfregierungen hin, wenn diese sich an TIRDEP-Personal richteten. ${ }^{196}$

Das Konzept der Partizipation war jedoch anschlussfähig für verschiedene Interpretationen, die auf älteren Ansätzen aufbauten. Bedienstete der finanziell anämischen tansanischen Behörden verstanden unter „Hilfe zur Selbsthilfe“ und self-reliance zunehmend nur noch, dass die Bevölkerung moralisch verpflichtet war, zum staatlichen Entwicklungsprojekt beizutragen und daher sämtliche finanziellen und Arbeitsleistungen selbst aufbringen sollte, während offizielle Stellen nur punktuelle Unterstützung leisteten. ${ }^{197}$ Diese Form der „Partizipation von oben“ war bereits im kolonialen community development etabliert und im Rahmen von Ujamaa-Diskursen aktualisiert worden. ${ }^{198}$ Erfahrene BeamtInnen der Tanga-Region sprachen sich aber gegen übersteigerte Erwartungen an die „Selbsthilfe“ aus: Da solche Maßnahmen oft mit Zwang und Drohmaßnahmen sowie der Erinnerung an die kolonialen Enwicklungsinterventionen verbunden waren, hätten sie geringe Erfolgschancen. ${ }^{199}$ Mitte der 1980er-Jahre kritisierten sie den Selbsthilfeansatz für die Langatmigkeit in der Umsetzung und die zu hohen Forderungen, die das TIRDEP-Projekt VDP an die Bevölkerung stellte - ein deutlicher Kontrast zu den zwei vorherigen Jahrzehnten. ${ }^{200}$

Der größte Widerstand gegen die Partizipation von oben kam jedoch aus den Dörfern selbst. Projekte mit „Selbsthilfekomponente“ stießen in vielen Dörfern auf dasselbe Problem: „low motivation on the villagers“ side to contribute through self-help activities““. ${ }^{201}$ Der Zeit- und Ergebnisdruck, unter dem die expatriates

196 Ngware/Nindi, Sociological Survey, S. 200: „[I]n order for such leadership to survive and actually continue to serve their personal interests, they cheated both the government and TIRDEP officials e.g. that the farmers had understood and agreed to take part“ - und das, obwohl keinerlei Diskussionen stattgefunden hatten.

197 Rebecca Marsland, Community Participation the Tanzanian Way: Conceptual Contiguity or Power Struggle? in: Oxford Development Studies 34/1 (2006), S. 65-79.

198 Irmi Maral-Hanak, Language, Discourse and Participation. Studies in Donor-Driven Development in Tanzania. Wien 2009, S. 22-41.

199 Tanga Library, [VDP Reports], VDP Project Manager, Project Progress Report No. 1, Tanga, 28.12.1981, S. 9.

200 Ebd., VDP Project Manager, Project Progress Report No. 12, Tanga, Juni 1987, S. 15.

201 Tanga Library, TIR PAM 0043, Regional Commissioner's Office Tanga and TIRDEP, Village Development Fund - Plan of Operation, Tanga, September 1980, S. 1; ebd., TL 49, TIRDEP Annual Report 1976, Tanga, März 1977, S. 2; BMZ, B 213/48198, Project Manager TIRDEP VWSP, Progress Report Number 7, Tanga, 22.12.1987, S. 10; Pilgram/Zils, TIRDEP, S. 81-82. Die Ursachen für die fehlende Bereitschaft, Arbeitsleistungen für das Projekt bereitzustellen sind äußerst heterogen und können hier nicht näher behandelt werden; darunter fallen u. a. notwendigere Subsistenztätigkeiten, Lohnarbeit für einen finanzstarken Unternehmer in der Umgebung, politische 
standen, gab den „Zielgruppen“, die über Jahrzehnte hinweg Erfahrung im Umgang mit Interventionsversuchen gesammelt hatten und auch relativ unabhängig von äußeren Ressourcen überlebten, dabei eine bessere Ausgangsposition:

Farmers [...] often withheld the self-help work, knowing that the project might pay them for it in the end, because projects have a time schedule and we cannot affor[d] to leave them half-finished. ${ }^{202}$

Sämtliche Stellen, die über mehr Macht in der Ressourcenallokation verfügten in der tansanischen Bürokratie, in den Reihen der GTZ und auch externe EvaluatorInnen - beklagten diesen pragmatischen Umgang als Ausdruck einer „Nehmermentalität““. ${ }^{203}$ Die materielle Potenz von TIRDEP wurde zum Bumerang, da gerade die Projekte mit Selbsthilfekomponente auf ihren Nimbus als effiziente und verlässliche Ressourcenbeschaffer angewiesen waren: „we should never be the bottleneck in project implementation, or we will face the danger the self-help work is withdrawn“, warnte ein GTZ-Experte einen Kollegen in einem anderen Projekt. ${ }^{204}$ Die entwicklungspolitischen Prämissen führten hier, zumindest was die gewünschten Projektergebnisse anging, auch zu einer höheren Abhängigkeit des Projekts von den „Zielgruppen“. In dieser Hinsicht übte das Partizipationsund Selbsthilferegime also auch eine disziplinierende Wirkung auf das Projektpersonal aus, das sich von den offiziellen Stellen und „Parteibonzen“ dadurch unterscheiden sollte, dass Versprechen für materielle Transfers an die Dörfer tatsächlich eingehalten wurden, wie sich ein ehemaliger GTZ-Experte sich im Interview erinnerte. ${ }^{205}$ Gerade dadurch verfestigte sich aber auch die Wahrnehmung von diesem Projekt (VDP) ,as ,a better state' and the ,image' of [...] TIRDEP as an impl[e]menting agency“, wie es später in einem internen Diskussionspapier hieß. ${ }^{206}$ Das VDP-Arrangement, in dem die Dörfer auch selbst Kostenbeiträge leisten sollten, wurde durch die von westlichen Gebern geforderten makroökonomischen Reformen zudem impraktikabel. Die Abwertung des tansanischen Schillings 1986 (gefolgt vom weiteren Wertverlust in den kommenden Jahren)

Auseinandersetzungen, generelles Misstrauen gegenüber den lokalen Autoritäten, aber auch die Erwartung, keinen Nutzen aus den jeweiligen Projekten zu ziehen, der dem Aufwand entsprechen würde.

202 Tanga Library, TIR PAM 0060, VDP Project Manager, Project Progress Report January-June 1982, Tanga, 30.6.1982, S. 13.

203 Ebd., [General Correspondences], Project Manager VDP an UNRSD, o.O., 9.11.1981.

204 Ebd., VDP an VWSP, Tanga, 18.6.1984.

205 Interview \#26, GTZ-Experte.

206 Tanga Library, [PFK '92 -Decentraliz, PRDVL, Reports], o.A., Discussion paper No. 1 (Second Draft), o. O., 28.1.1992. 
machte den Projektansatz, Geld und Naturalien aus den Dörfern als Zahlung für Importe zu verrechnen mit einem Schlag unattraktiv: Die Austauschrelationen hatten sich derart zum Nachteil der Dörfer entwickelt, dass die Beteiligung an den Projektkosten nun kaum noch leistbar war. ${ }^{207}$ Damit wurde das deutsche TIRDEPPersonal umso mehr in die ungeliebte Rolle als Ressourcenbeschaffer gedrängt.

\section{Fazit}

TIRDEP ist damit Ausdruck und Motor einer sich wandelnden Dynamik in den Beziehungen zwischen „Gebern“ und „Nehmern“, zwischen Staat und StaatsbürgerInnen, zwischen expatriates und Counterparts, zwischen Projektpersonal und Zielgruppen. Relativ konstant blieb jedoch, dass TIRDEP eine Art „Parallelregierung“ in Tanga bildete und westdeutsche EntscheidungsträgerInnen in der Ressourcenallokation das letzte Wort behielten: selbst wenn die Machtverhältnisse in vielen lokalen Arenen polyzentrisch waren, blieben die Makrostrukturen doch durch ein deutliches Machtgefälle gekennzeichnet. In vielerlei Hinsicht boten die Projekte von TIRDEP für die beteiligten TansanierInnen die Möglichkeit, sich Ressourcen anzueignen und lokale Engpässe zu überwinden. ${ }^{208}$ Besonders offensichtlich wird das mit dem Blick auf die räumlichen, personellen, aber auch zeitlichen Ränder der Projekte. Die Intensität der Aktivitäten, die von tansanischen Behörden nach dem „Handover“ weitergeführt werden sollten, ging in der Regel genauso zurück wie Möglichkeiten der einzelnen tansanischen ExpertInnen, ihre Kenntnisse anzuwenden. Tansanische Interviewpartner sahen dabei nicht die von vornherein überdimensionierte Anlage von Projektaktivitäten, sondern die Ressourcenschwäche des Staates als ausschlaggebend an:

They had a lot of motorcycles, cars, which also cost a lot of maintenance, the allowances for the workers. So when the project comes to an end, you can see some of the good things are coming down because of the sustainability of the funding. ${ }^{209}$

207 Vgl. auch BMZ, B 213/48198, RWD Heft 6, VWSP Progress Report No. 6, Tanga, 30.6.1987, S. 9; BMZ, B 213/48209, VWSP Project Progress Report No. 10, Tanga, 16.6.1989, S. 17; zu SECAP siehe z.B. Michael Mdoe, The Impact of Rangeland Rehabilitiation in Mbwei Village in the West Usamabara Mountains of Tanzania: Seven Years Experience in Mbwei Village, Lushoto 1989, S. 15. 208 Siehe hierzu auch Green, The Development State.

209 Interview \#16, Tansanischer Entwicklungsplaner; siehe auch Interview \#7, Tansanischer Entwicklungsplaner und Counterpart. 
Obwohl die expatriates verhindern wollten, Parallelstrukturen und verschiedene „Gehaltsklassen“ zu etablieren, ist genau das in vielen Fällen geschehen. Nur die wenigsten Projektaktivitäten wurden fortgeführt; tansanische Beteiligte mussten Wege finden, um den Übergang in den „Normalzustand“ (hali halisi) ohne Nebeneinkünfte wie Tagessätze (posho) zu gestalten - oder die Extraversionsstrategie mithilfe anderer Geber fortzusetzen, die ab Ende der 1980er Jahre in Tanga wieder aktiver wurden und der bundesdeutschen Dominanz in der Region ein Ende bereiteten. ${ }^{210}$ Die Dynamiken und Prozesse in TIRDEP sind aber nicht in Isolation oder in einfachen kausalen Schemata erklärbar; sie zeigen vielmehr, wie in der Entwicklungsarbeit lokale, nationale und globale Ebenen von Disziplinierung miteinander verflochten waren. Das Aufkommen der per diem-Kultur ${ }^{211}$ war u.a. eine Reaktion auf die sozialen Auswirkungen der Wirtschaftskrise, entwickelte dann aber ein bis heute wirksames Eigenleben. Die Einführung partizipativer Methoden koinzidierte mit der zunehmenden Qualifikation der Counterparts, während die Hoheit über Konzepte und Ressourcenallokationen größeren Ausmaßes aber auf Geberseite verblieb. Die kommunale Eigenverantwortung für Infrastruktur und die Betonung der „Hilfe zur Selbsthilfe“ wiederum war die Kehrseite zum Um- und Rückbau des Entwicklungs- und Wohlfahrtsstaates und dem zunehmenden Vertrauensverlust, den der Staat bei Gebern und in der Bevölkerung erlitten hatte. Der Doppeldiskurs von neoliberaler Sparpolitik und good governance warf in dieser Abkehr von den Institutionen des Entwicklungsstaates bereits seine Schatten voraus.

Die Interdependenz der Counterpartbeziehungen in der „Arena“ TIRDEP zeigte sich darin, dass sich expatriates zu Maßnahmen gezwungen sahen, die nicht entwicklungspolitisch legitimierbar waren, aber die Aufrechterhaltung des modus vivendi mit den lokalen Eliten garantierten. ${ }^{212}$ Deren politisches und institutionelles Kapital, ihre Entscheidungsbefugnisse und sozialen Netzwerke waren für die Projektumsetzung häufig unverzichtbar; nicht zuletzt waren die Maßnahmen auch von tansanischen Leistungen, materiell wie personell, abhängig. Die expatriates mussten sich an die „Spielregeln“ halten, sich mit Counterparts arrangieren und auf die Konten der „Vertrauensbanken“213 einzahlen,

210 Interview \#56, Tansanischer Landwirtschaftsexperte, Counterpart und späterer Projektmanager.

211 Daniel Jordan Smith, Patronage, Per Diems and the „Workshop Mentality“: The Practice of Family Planning Programs in Southeastern Nigeria, in: World Development 31/4 (2003), S. 703715.

212 Das Prinzip lautete: „Eine Hand wäscht die andere“. Interview \#28, GTZ-Projektleiter. Siehe für ein weiteres Beispiel auch Jennings, Surrogates of the State, S. 194.

213 Hüsken, Stamm der Experten. 
um bei Bedarf Wohlwollen ausgezahlt zu bekommen und ihren Handlungsspielraum aufrechtzuerhalten. Die Machtverhältnisse waren also vielgestaltig, kontextabhängig und bisweilen instabil - allerdings nur auf der Mikro- und bisweilen auf der Meso-Ebene; nicht auf der Makro-Ebene.

Obwohl die Beziehungen zwischen Projektpersonal, Verwaltung, Partei und „Zielgruppen“ von einer (gerade im ökonomischen Bereich offensichtlichen) Machtungleichheit geprägt waren, war der Ausgang einzelner Aushandlungsprozesse keineswegs vorbestimmt. Die ökonomische Stärke konnte im Fall von „Selbsthilfe“-Projekten mit ihren voraussetzungsreichen Vorannahmen zur Schwäche werden. Die Counterpartbeziehungen und Beziehungen zu weiteren Beteiligten waren zwar asymmetrisch, aber da sie auch interdependent waren, blieben Spielräume auf allen Seiten bestehen. DorfbewohnerInnen etwa kamen an Leistungen, ohne die „Selbsthilfebeiträge“ vollständig zu leisten, und die Disziplinierungsmaßnahmen wurden vom staatlichen Personal immer wieder unterlaufen, gerade dann, wenn keine persönlichen Vorteile aus der Arbeit mit den TIRDEP-Projekten gezogen werden konnte.

Zeit- und Ergebnisdruck sowie Karriereerwartungen und Rollenbilder führten das GTZ-Personal dabei immer wieder zu paternalistischen Handlungsweisen, selbst wenn sie „Besserwisserei“ und „Alleingänge“ aus Überzeugung ablehnten. Die Widersprüche und unintendierten Konsequenzen der Entwicklungsarbeit waren damit am eigenen Paternalismus wider Willen erfahrbar. Während dieser Paternalismus eine Konstante der Entwicklungsarbeit mit ihren Ungleichheitsbeziehungen darstellt, lässt sich ein historischer Wandel in der Tatsache beobachten, dass wachsende soziale Ungleichheit - nicht nur zwischen expatriates und Counterparts, sondern selbst auf Ebene konkreter Dorfgemeinschaften - in den 1980er Jahren zunehmend als normal oder zumindest nicht bearbeitbar erachtet wurde. Auch das hatte der Wandel vom top-down- zum bottom-up-Ansatz mit sich gebracht. Vom Anspruch, mit dem eigenen Handeln die Verhältnisse zu ändern, blieb wenig mehr als dessen Umkehrung: nämlich die mageren Ergebnisse des Handelns mit den ungünstigen Verhältnissen zu erklären. 